University of Wollongong

Research Online

Australian Institute for Innovative Materials -

Papers

Australian Institute for Innovative Materials

$1-1-2019$

Functionalizing graphene with titanate coupling agents as reinforcement

for one-component waterborne poly(urethane-acrylate) anticorrosion

coatings

\author{
Haihua Wang \\ Northwestern University \\ Yu He \\ Shaanxi University of Science and Technology \\ Guiqiang Fei \\ Shaanxi University of Science and Technology \\ Caiyun Wang \\ University of Wollongong, caiyun@uow.edu.au \\ Yiding Shen \\ Shaanxi University of Science and Technology
}

See next page for additional authors

Follow this and additional works at: https://ro.uow.edu.au/aiimpapers

Part of the Engineering Commons, and the Physical Sciences and Mathematics Commons

Research Online is the open access institutional repository for the University of Wollongong. For further information contact the UOW Library: research-pubs@uow.edu.au 


\title{
Functionalizing graphene with titanate coupling agents as reinforcement for one- component waterborne poly(urethane-acrylate) anticorrosion coatings
}

\author{
Abstract \\ Achieving a uniform dispersion of reduced graphene oxide (RGO) nanosheets in waterborne polymer \\ matrix remains a challenge. Here, we significantly improved the dispersion stability of RGO in a polymer \\ matrix through functionalizing RGO with titanate coupling agent of different dendritic structure. Titanate \\ coupling agents of two branched dioctylpyrophosphate (T2) and three branched dioctylpyrophosphate \\ (T3) were used. Rather than simply blending graphene with polymer matrix, functionalized graphene \\ (T2-RGO or T3-RGO) was introduced into the reaction system with monomers to participate in the \\ polymerization; resulting in improved compatibility and interaction between the graphene and polymer, \\ especially for T3-RGO. One-component waterborne poly (urethane-acrylate) nanocomposite coatings \\ (WPUA/T2G or WPUA/T3G) were then obtained. The particle size of WPUA/T3G colloidal particle size is \\ much smaller than that of WPUA/T2G, the colloidal stability was also increased with the incorporation of \\ T3-RGO. Compared with pure WPUA, the tensile strength of WPUA/T3G nanocomposite increased from \\ 17.78 MPa to $40.01 \mathrm{MPa}$, and the elongation at break increased from $249 \%$ to $424 \%$. The tensile strength \\ and elongation at break of WPUA/T2G were $32.01 \mathrm{MPa}$ and $366 \%$, which were inferior to that of WPUA/ \\ T3G. Moreover, in comparison with pure WPUA, the impedance modulus of WPUA/T3G increased from \\ $1.12 \times 105 \Omega . c m 2$ to $1.15 \times 108 \Omega . c m 2$, the coating resistance of WPUA/T3G increased from $8.41 \times 104$ \\ $\Omega . c m 2$ to $1.39 \times 108 \Omega . c m 2$, which was also much higher than the impedance modulus $(6.93 \times 106$ \\ $\Omega . c m 2)$ and coating resistance $(7.31 \times 106 \Omega . \mathrm{cm} 2)$ of WPUA/T2G. WPUA/T3G nanocomposite coating \\ exhibited excellent long-term corrosion resistance, being superior to the previously reported performance \\ of solventborne polyurethane/graphene composite. \\ Disciplines \\ Engineering | Physical Sciences and Mathematics

\section{Publication Details} \\ Wang, H., He, Y., Fei, G., Wang, C., Shen, Y., Zhu, K., Sun, L., Rang, N., Guo, D. \& Wallace, G. G. (2019). \\ Functionalizing graphene with titanate coupling agents as reinforcement for one-component waterborne \\ poly(urethane-acrylate) anticorrosion coatings. Chemical Engineering Journal, 359 331-343.

\section{Authors} \\ Haihua Wang, Yu He, Guiqiang Fei, Caiyun Wang, Yiding Shen, Ke Zhu, Liyu Sun, Nana Rang, Danhui Guo, \\ and Gordon G. Wallace
}




\section{Functionalizing graphene with titanate coupling agents as reinforcement for one-component waterborne poly(urethane-acrylate) anticorrosion coatings}

Haihua Wang ${ }^{\mathrm{a}, \mathrm{b}, *}, \mathrm{Yu} \mathrm{He}^{\mathrm{a}}$, Guiqiang Fei ${ }^{\mathrm{a}, *}$, Caiyun Wang ${ }^{\mathrm{c}}$, Yiding Shen ${ }^{\mathrm{a}}, \mathrm{Ke} \mathrm{Zhu}^{\mathrm{a}}$, Liyu Sun ${ }^{\mathrm{a}}$, Nana Rang ${ }^{\text {a }}$, Danhui Guo ${ }^{\mathrm{a}}$, and Gordon G. Wallace ${ }^{\mathrm{c}}$

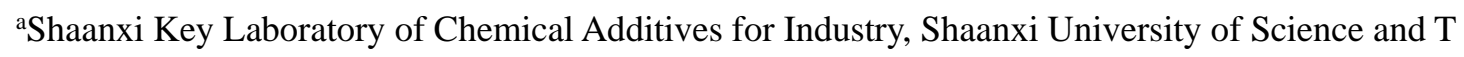
echnology, Xi'an 710021, China

${ }^{b}$ Department of Material Science and Engineering, Northwestern University, Evanston, Illinois 60208, USA

'Intelligent Polymer Research Institute, ARC Centre of Excellence for Electromaterials Science, AIIM Facility, University of Wollongong, NSW 2500, Australia.

ABSTRACT: Achieving a uniform dispersion of reduced graphene oxide (RGO) nanosheets in waterborne polymer matrix remains a challenge. Here, we significantly improved the dispersion stability of RGO in a polymer matrix through functionalizing RGO with titanate coupling agent of different dendritic structure. Titanate coupling agents of two branched dioctylpyrophosphate (T2) and three branched dioctylpyrophosphate (T3) were used. Rather than simply blending graphene with polymer matrix, functionalized graphene (T2-RGO or T3-RGO) was introduced into the reaction system with monomers to participate in the polymerization; resulting in improved compatibility and interaction between the graphene and polymer, especially

*Corresponding author at: Shannxi Key Laboratory of Chemical Additives for Industry, Shannxi University of science and Technology,Xi,an 710021,China

E-mail address: whh@sust.edu.cn (Haihua Wang); feiguiqiang@sust.edu.cn (Guiqiang Fei) 
for T3-RGO. One-component waterborne poly (urethane-acrylate) nanocomposite coatings (WPUA/T2G or WPUA/T3G) were then obtained. The particle size of WPUA/T3G colloidal particle size is much smaller than that of WPUA/T2G, the colloidal stability was also increased with the incorporation of T3-RGO. Compared with pure WPUA, the tensile strength of WPUA/T3G nanocomposite increased from 17.78 $\mathrm{MPa}$ to $40.01 \mathrm{MPa}$, and the elongation at break increased from $249 \%$ to $424 \%$. The tensile strength and elongation at break of WPUA/T2G were $32.01 \mathrm{MPa}$ and $366 \%$, which were inferior to that of WPUA/T3G. Moreover, in comparison with pure WPUA, the impedance modulus of WPUA/T3G increased from $1.12 \times 10^{5} \Omega . \mathrm{cm}^{2}$ to $1.15 \times 10^{8} \Omega . \mathrm{cm}^{2}$, the coating resistance of WPUA/T3G increased from $8.41 \times 10^{4}$ $\Omega . \mathrm{cm}^{2}$ to $1.39 \times 10^{8} \Omega . \mathrm{cm}^{2}$, which was also much higher than the impedance modulus $\left(6.93 \times 10^{6} \Omega . \mathrm{cm}^{2}\right)$ and coating resistance $\left(7.31 \times 10^{6} \Omega . \mathrm{cm}^{2}\right)$ of WPUA/T2G. WPUA/T3G nanocomposite coating exhibited excellent long-term corrosion resistance, being superior to the previously reported performance of solventborne polyurethane/graphene composite.

Keywords: Titanate coupling agent, Graphene, Polyurethane, Corrosion, Coating

\section{Introduction}

Protective organic coatings are the most common and effective method to protect metals owing to their advantages of low cost, scalability and easy controllability [1]. Among them, waterborne coatings have become the predominant development trend due to environmental and human health issues. Waterborne polyurethane (WPU) 
coating is one of the most important coatings due to its high flexibility, excellent chemical resistance and abrasion resistance, as well as good adhesion. Organic coating can provide a physical barrier between the corrosive medium and metal substrate [2]. However, the low resistance of WPU against external stress easily creates defects in the nanocomposite coating, which would facilitate the penetration of corrosive medium [3-4]. Additionally, the cross-linking density of coating declines after immersing in corrosive medium, leading to the decrease of adhesion and coating deterioration [2].

Incorporating nanofillers into polyurethane coatings has been demonstrated as an effective approach to improve the corrosion resistance of organic coatings via increasing the electrolyte pathway length [5-11]. Graphene, which comprises a single-atom-thick crystal of $\mathrm{sp}^{2}$-bonded carbon, is regarded as a promising candidate for anti-corrosive nanofiller and has been largely reported and used for metal anticorrosion [12-14]. However, different from graphene oxide (GO), graphene nanosheets have a high tendency to agglomerate in the polymer matrix even at a very low loading. The agglomeration decreases the compatibility and interactions between graphene and the polymer matrix, and thereby impairs the corrosion resistance of the nanocomposite coatings [15-17]. Moreover, it has been reported that curing reaction could be retarded due to physical hindrance generated by nanofiller even at lower concentration [18]. The presence of reactive functional groups on the surface of graphene nanosheets and the homogeneous dispersion of graphene in polymer nanocomposite are beneficial to speed up the cure reaction of polymer/graphene nanocomposite [19-20]. Moreover, the functional groups on the graphene surface can act as active sites for bonding polymer matrix and subsequently improve the 
compatibility of polymer nanocomposite.

Heretofore, non-covalent and covalent functionalization of graphene nanosheets has been adopted to improve the dispersion of graphene and impede the re-stacking of graphene in polymer matrix [20-24]. For instance, surfactant, dispersant, larger aromatic stabilizer [20-22], silane coupling agent [23-25] and titanate coupling agent $[14,26]$ have been adopted to prepare functionalized graphene. Li et al certified that titanate-functionalized reduced GO (RGO) can facilitate the dispersion of graphene WPU in composite coating, but some blister was observed after salt spray test for $48 \mathrm{~h}$ [14]. However, submicron-sized agglomeration also took place in titanate-functionalized RGO/PU composite when RGO and WPU are simply blended since the agglomerated graphene would result in isolated "islands" of graphene, instead of the barrier network [26]. Therefore, the uniform dispersion of RGO in waterborne polymer coatings is still the main challenge, as well as the formation of a continuous barrier network in polymer matrix, since the vast interfacial area created by well-dispersed nanoparticles can significantly affect the performance of polymer nanocomposite [27].

In this study, two novel titanate coupling agents of different dendritic structures, such as bis(P,P-bis-ethylhexyl diphosphato) ethanediolato titanate (T2) and isopropyl tri(diotylpyrophosphate) titanate (T3), were adopted to functionalize RGO. To our best knowledge, there are few researches on the T2 and T3 functionalized RGO. The main motivation of this research is to improve the uniform dispersion of RGO in waterborne poly (urethane-acrylate) (WPUA) coating and build superb polymer-graphene interactions in nanocomposite coating through the incorporation of 
novel titanate coupling agents. Also, a systematic and comparative investigation on the effects of dendrimer structure on coating performance was also performed to further understand the structure-property relationship.

Rather than physically blending titanate-functionalized RGO (T2-RGO and T3-RGO) and WPUA, T2-RGO and T3-RGO were mixed with reaction monomers during the preparation process of $-\mathrm{NCO}$ terminated polyurethane prepolymer via the in-situ surfactant-free emulsion polymerization based on our previous study [28]. This facile method is able to further improve the uniform dispersion and compatibility of RGO in WPUA matrix, and chemical bonds can be formed between -NCO terminated polyurethane prepolymer and hydroxyl groups on T2-RGO and T3-RGO, which is favorable to prominently improve the compatibility and interaction between RGO and WPUA, and simultaneously promote the nanoscopic dispersion of RGO in WPUA. The as-prepared WPUA/RGO nanocomposite coatings showed superior tensile strength, elongation at break and corrosion resistance. This work provides a novel approach to the realization of a uniform dispersion of RGO in waterborne polymer matrix and improves the stability of waterborne polymer/graphene dispersions, as well as to fabricate one-component anticorrosion coatings.

\section{Experimental section}

\subsection{Materials}

Bis(P,P-bis-ethylhexyl diphosphato) ethanediolato titanate (T2) and isopropyl tri(diotylpyrophosphate) titanate (T3) were purchased from Nanjing PinNing Coupling Agent Co. Ltd. Natural flake graphite with an average particle size of $45 \mu \mathrm{m}$ (99\%) was purchased from Qingdao Haida Graphite Co. Ltd. Concentrated sulfuric 
acid $\left(\mathrm{H}_{2} \mathrm{SO}_{4}, 98 \%\right)$, concentrated hydrochloric acid $(\mathrm{HCl})$, sodium nitrate $\left(\mathrm{NaNO}_{3}\right)$, hydrogen peroxide $\left(\mathrm{H}_{2} \mathrm{O}_{2}\right)$, and potassium permanganate $\left(\mathrm{KMnO}_{4}\right)$ were obtained from Tianjin Kemiou Chemical Reagent Co. Ltd. Hydrazine hydrate (80\%, analytically pure) was from Tianjin Fuyu Fine Chemical Co. Ltd. Ammonia $\left(\mathrm{NH}_{3} \cdot \mathrm{H}_{2} \mathrm{O}\right)$, hydroxyethyl methacrylate (HEMA), methyl methacrylate (MMA), butyl acrylate (BA), triethylamine (TEA), ammonium persulfate (APS), and glycidyl methacrylate (GMA) were purchased from Tianjin Chemical Reagent Co. Ltd. Isophorone diisocyanate (IPDI), polycaprolactone diol (PCL1000) and dimethylolbutanoic acid (DMBA) were purchased from Taisen Chemical. Trimethylolpropane (TMP) was purchased from Shanghai Haiyu Chemical Co. Ltd. Alpha alkenyl sulfonate (AOS) was obtained from Hubei Haiyi Pharmaceutical Co. Ltd.

2.2. Synthesis of reduced graphene (RGO) and Functionalized Graphene.

Graphite oxide (GO) was prepared by the modified Hummers method as we previously reported [29]. GO dispersion $(1 \mathrm{mg} / \mathrm{ml})$ was placed in a three-necked flask and sonicated for $30 \mathrm{~min}$ using an ultrasonic bath sonicator. Then the flask was transferred to a water bath, the $\mathrm{pH}$ value was adjusted to 10 , followed by the addition of $\mathrm{T} 2$ or $\mathrm{T} 3$. The reaction temperature for $\mathrm{T} 2$ and $\mathrm{T} 3$ was $90{ }^{\circ} \mathrm{C}$ and $70{ }^{\circ} \mathrm{C}$, respectively. After $3 \mathrm{~h}, 0.3 \mathrm{~g}$ of hydrazine hydrate was added and the reaction was kept for another $5 \mathrm{~h}$. T2 functionalized graphene (T2-RGO) and T3 functionalized graphene (T3-RGO) were thereby obtained via filtration and rinsed with deionized water until the $\mathrm{pH}$ value reached 7 , and dried at $60{ }^{\circ} \mathrm{C}$ in a vacuum oven for $48 \mathrm{~h}$. 
RGO was prepared based on the above-mentioned process without the addition of T2 and $\mathrm{T} 3$, except that the final step for the reduction reaction with hydrazine hydrate was performed at $85^{\circ} \mathrm{C}$ for $8 \mathrm{~h}$.

\subsection{Preparation of Waterborne Poly(urethane-acrylate)/Functionalized Graphene} Nanocomposite Emulsions.

PCL1000 (10 g), IPDI (7 g), DMBA (1.8 g), TMP (0.3 g), MMA (4 g), BA (1 g) and different amount of T2-RGO or T3-RGO $(0 \%, 0.1 \%, 0.3 \%, 0.5 \%, 0.7 \%, 0.9 \%)$ were add to a dry three-necked flask under constant stirring. The reaction temperature was increased to $75^{\circ} \mathrm{C}$ after stirring for $1 \mathrm{~h}$, and the reaction was kept for another $2 \mathrm{~h}$ to prepare polyurethane prepolymer. Then $1.56 \mathrm{~g}$ of HEMA was added to endcap NCO-terminated polyurethane prepolymer, followed by the neutralization with trimethylamine. The molar ratio of trimethylamine to DMBA is $1: 1$. After $20 \mathrm{~min}$, AOS aqueous solution was added dropwise to generate the phase inversion process over a period of $30 \mathrm{~min}$, followed by the addition of ammonium persulfate $(0.2 \mathrm{~g})$ to initiate the reaction among vinyl monomers. Simultaneously the mixture of $10.4 \mathrm{~g}$ MMA and 2.6 g BA was added. Waterborne poly (urethane-acrylate)/functionalized graphene nanoemulsions were thereby obtained after the reaction for another $2 \mathrm{~h}$.

\subsection{Preparation of Nanocomposite Films.}

Nanoemulsions were casted on a tetrafluoroethylene plate and left at room temperature for $48 \mathrm{~h}$, followed by a drying process at $60{ }^{\circ} \mathrm{C}$ in a vacuum oven for $48 \mathrm{~h}$. The film could be easily peeled off and kept in a desiccator to avoid the absorption of moisture. 


\subsection{Preparation of Nanocomposite Coatings.}

Nanoemulsions were blade-coated on the tinplate to prepare environmental anticorrosive coatings according to GB/T1727.92.

\subsection{Characterizations.}

Phase structure was analyzed by a D/max2200PC X-ray powder diffractometer (XRD, Rigaku) equipped with $\mathrm{CuK} \alpha$ radiation. Molecular structure was determined by a Renishaw-invia micro-confocal laser Raman spectrometer. Micromorphology was observed by a Tecnai G2 F20 S-TWIN transmission electron microscope (TEM, FEI) and an S-4800 scanning electron microscope (SEM, Hitachi). Microstructure was observed using a SPA400-SPI3800N atomic force microscope (AFM, Seiko) in the tapping mode. Thermal behavior was investigated using a US TA TGQ500 thermogravimeter at a heating rate of $10^{\circ} \mathrm{C} / \mathrm{min}$ over a temperature range of 20 to $600{ }^{\circ} \mathrm{C}$

Particle size of the nanoemulsions was measured by use of a Malvern Mastersizer 2000 particle size analyzer. The dispersion stability was analyzed by a dispersion stabilizer (Formulaction, Turbiscan Lab) at room temperature at a scanning rate of $54 \mathrm{~s}^{-1}$. The water absorption was calculated according to the reported method [30]. Contact angle was determined by a KRÜSS DSA-100 contact angle meter. Tensile strength was evaluated on a TS2000-S universal testing machine (Scientific and Technological Limited Co., High Iron). The measurements were repeated by five times.

Salt spray test was performed on a HJ-YW60 salt spray test machine (Dongguan 
Hengjun Instrument Equipment Co., Ltd.) according to GB/T1771-2007. EIS spectra analyses were carried out in a 3.5 wt. $\% \mathrm{NaCl}$ aqueous solution at room temperature by using a PARSTAT MC 2000 multi-channel electrochemical testing system (AMETEK). A three-electrode cell configuration was used. Sample with an exposed area of $1 \mathrm{~cm}^{2}$ was used as working electrode coupled with a calomel reference electrode and a platinum foil counter electrode $\left(1 \mathrm{~cm}^{2}\right)$. The electrochemical measurements were performed on three replicates to ensure the repeatability of data.

\section{Results and discussion}

\subsection{Preparation, Stability and Microstructure of Functionalized RGO}

RGO was functionalized with titanate coupling agents with different dendritic structure to improve the dispersibility in different solvents, and the preparation scheme is shown in Figure 1A. T2 and T3 titanate coupling agents were firstly utilized to react with the hydroxyl groups on the GO surface, followed by reduction reaction. Afterwards, partially reduced RGO functionalized with T2 and T3 molecules was obtained. The molecular structure of T2 and T3 is shown in Figure 1B; T3 possesses a higher degree of branching in comparison with T2. The morphology of T2-RGO and T3-RGO prepared with different mass ratios of GO to titanate coupling agents was investigated by TEM measurement. Aggregation state was detected for T2-RGO, while T3-RGO displayed a transparent and thin layered structure, which can be attributed to a higher degree of exfoliation induced by the intercalation of $\mathrm{T} 3$ with higher branching degree (Figures 1C, 1D). It was also found that aggregation behavior 
was weakened with the decreased ratio of GO to titanate coupling agent from 1:6 to $1: 7$, and became prominent when the ratio decreased further to $1: 8$. This phenomenon suggests that the titanate coupling agent is capable of exfoliating RGO nanosheets at an appropriate ratio; whereas excessive coupling agent cannot effectively exfoliate RGO sheets.

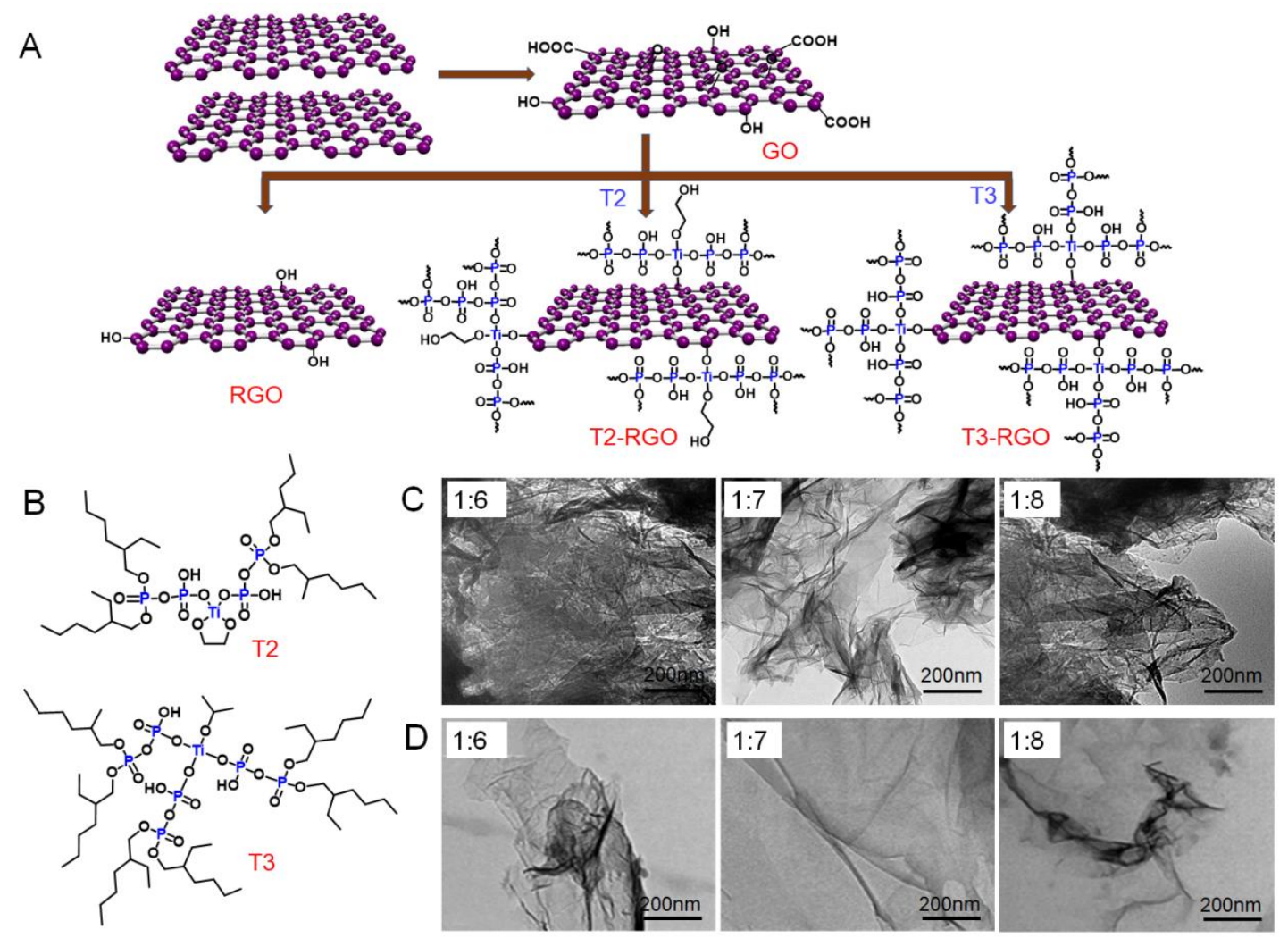

Figure 1. (A) Preparation schemes of RGO and RGO functionalized with different titanate coupling agents; (B) Chemical structure of titanate coupling agents (T2, bis (dioctyl pyrophosphate) ethylene titanate; T3, isopropyl tri (dioctylpyrophosphate) titanate); (C) TEM images of T2-RGO graphene and (D) T3-RGO graphene prepared with different mass ratios of graphene oxide to titanate coupling agents. 

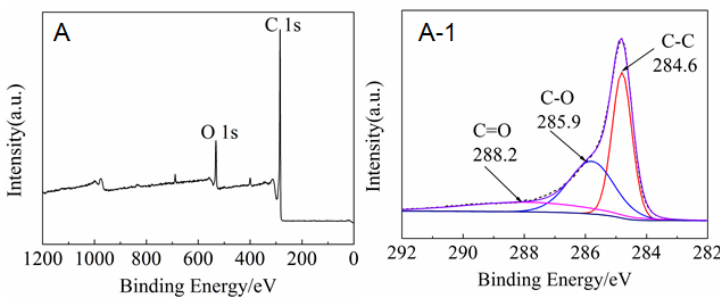

E
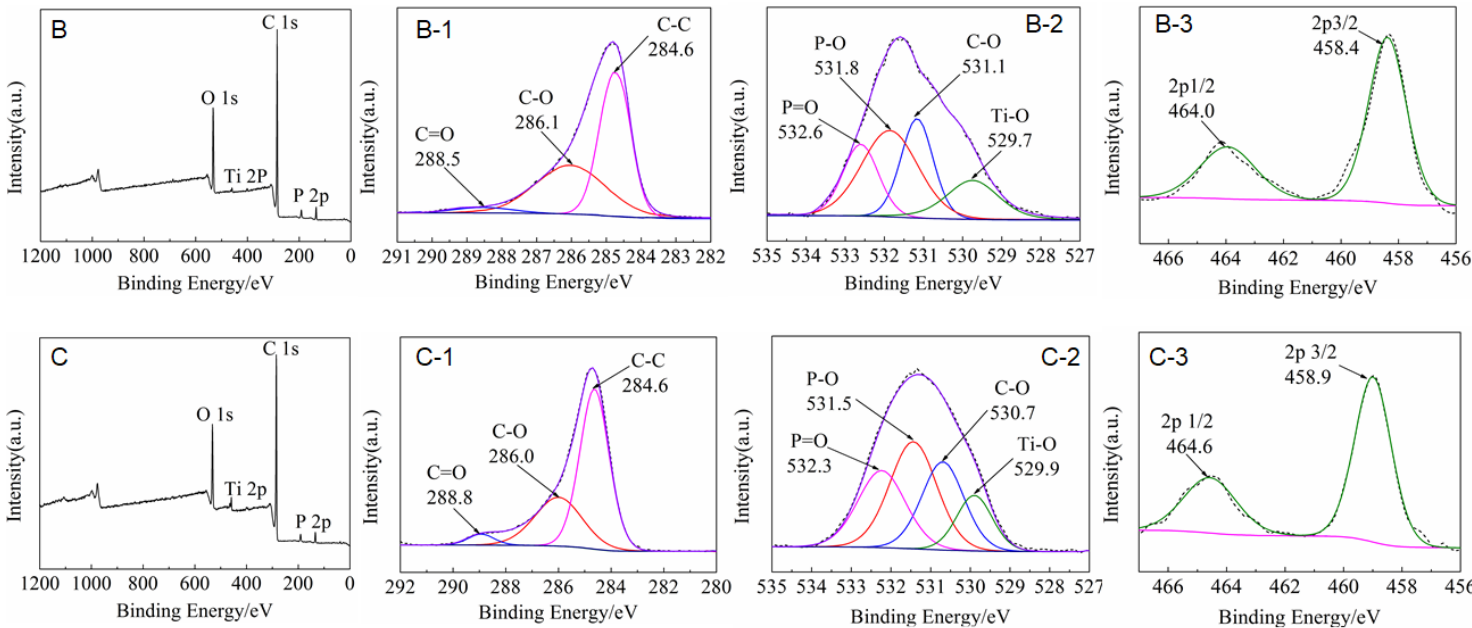

Figure 2. (A) XPS survey spectrum and (A-1) XPS C1s spectrum of RGO; (B) XPS survey spectrum, (B-1) XPS C1s spectrum, (B-2) O1s spectrum and (B-3) Ti2p spectrum of T2-RGO; (C) XPS survey, (C-1) XPS C1s spectrum, (C-2) O1s spectrum and (C-3) Ti2p spectrum of T3-RGO; (E) Elemental composition of RGO, T2-RGO and T3-RGO.

XPS spectra of RGO, T2-RGO and T3-RGO were illustrated in Figure.2. XPS survey spectra certified the presence of $\mathrm{P}$ and Ti elements on the RGO surfaces, the elemental composition is summarized in Figure.2E. In the high resolution $\mathrm{C} 1$ s spectra of RGO, three peaks at $284.6 \mathrm{eV}, 285.9 \mathrm{eV}$ and $288.2 \mathrm{eV}$ are observed, which are corresponding to $\mathrm{sp}^{2}$ carbon, $\mathrm{sp}^{3}$ carbon/C-O and $\mathrm{C}=\mathrm{O}$, respectively [31]. This phenomenon indicated that partially reduced RGO was obtained. The high resolution $\mathrm{O}$ 1s spectra and Ti $2 \mathrm{p}$ spectra of T2- RGO and T3-RGO were also provided. The $\mathrm{O}$ 1s peaks at $529.7 \mathrm{eV}, 531.1 \mathrm{eV}, 531.8 \mathrm{eV}$ and $532.6 \mathrm{eV}$ are ascribed to Ti-O, C-O, P-O and $\mathrm{P}=\mathrm{O}$, respectively. Ti $2 \mathrm{p}_{1 / 2}$ peak centered at $464.0 \mathrm{eV}$ and $\mathrm{Ti} 2 \mathrm{p}_{3 / 2}$ peak centered at 
$458.4 \mathrm{eV}$ were detected in the Ti 2p XPS spectra [32]. The XPS survey spectra, together with $\mathrm{O} 1 \mathrm{~s}$ and $\mathrm{Ti} 2 \mathrm{p}$ spectra, demonstrated the successful surface functionalization of RGO with titanate coupling agent.
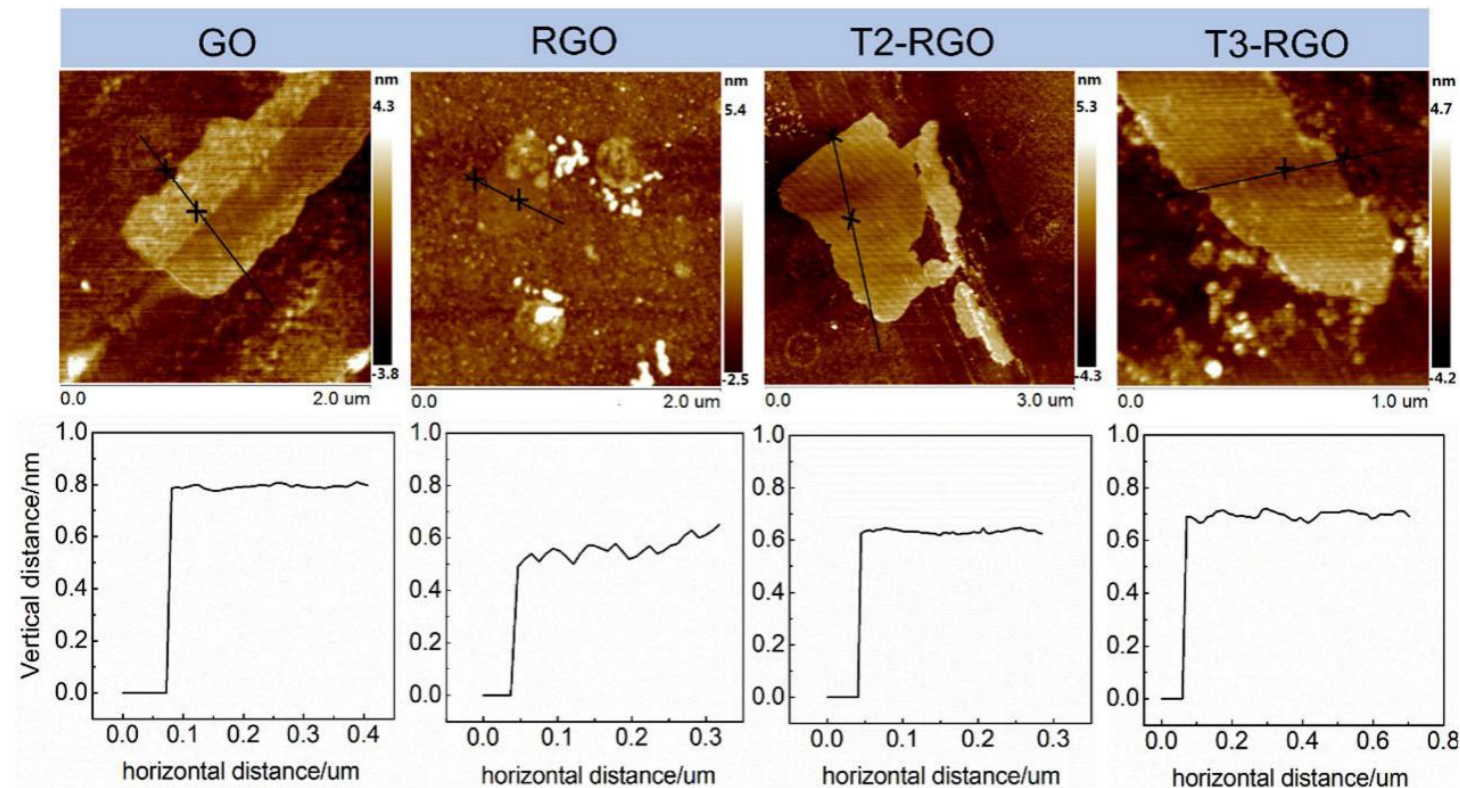

Figure 3. AFM images (upper) and height profiles (bottom) of GO, RGO, T2-RGO and T3-RGO.

AFM images and height profiles of GO, RGO, T2-RGO and T3-RGO nanosheets indicate that the average height was $0.786 \mathrm{~nm}, 0.583 \mathrm{~nm}, 0.625 \mathrm{~nm}$ and $0.692 \mathrm{~nm}$, respectively (Figure 3). In general, the thickness of a monolayer graphene is $0.34 \mathrm{~nm}$ [14]. In comparison with T2-RGO, the increased height of T3-RGO indicates the increased interlayer spacing between T3-RGO nanosheets due to the intercalated T3 of higher branching degree. It is also worthy to note that the nanosheet size of T2-RGO and T3-RGO was much bigger than that of RGO. This phenomenon suggests that the incorporation of titanate coupling agent was able to protect RGO nanosheets and avoid the breakdown of these nanosheets. The TEM and AFM results certified enlarged interlayer spacing between RGO nanosheets and larger RGO nanosheet size can be obtained with the incorporation of T3 with higher branching degree, which is 
beneficial to improving the barrier properties towards oxygen, water and electrolyte.

XRD patterns of GO, RGO, T2-RGO and T3-RGO nanosheets were also obtained, as shown in Figure 4A. GO presented a typical sharp peak at $2 \theta=10.8^{\circ}$, showing a highly ordered structure with interlayer spacing of $0.819 \mathrm{~nm}$. The diffraction peaks of RGO, T2-RGO and T3-RGO nanosheets were located at 23.9 , $22.3^{\circ}$ and $19.1^{\circ}$, respectively. The corresponding interlayer spacing was calculated to be $0.372 \mathrm{~nm}, 0.406 \mathrm{~nm}$ and $0.471 \mathrm{~nm}$. These results clearly demonstrated that the interlayer spacing was increased by the intercalation of titanate coupling agents and their branching degree as well.

Characteristic D band at around $1350 \mathrm{~cm}^{-1}$ which corresponds to the disordered structure $\left(\mathrm{sp}^{3}\right)$ to and $\mathrm{G}$ band at around $1580 \mathrm{~cm}^{-1}$ which corresponds to the ordered structure $\left(\mathrm{sp}^{2}\right)$ were observed in the Raman spectra of GO, T2-RGO and T3-RGO (Figures 4B, 4C and 4D) [33]. Their intensity ratios of $\mathrm{D}$ band to $\mathrm{G}$ band, $\mathrm{I}_{\mathrm{D}} / \mathrm{I}_{\mathrm{G}}$ values, were calculated to be $0.96,1.23$ and 1.34 for GO, T2-RGO and T3-RGO, respectively. $\mathrm{I}_{\mathrm{D}} / \mathrm{I}_{\mathrm{G}}$ ratio can be used to estimate the $\mathrm{sp}^{2}$ domain size and degree of disorder of graphene-based materials, where an increased $\mathrm{I}_{\mathrm{D}} / \mathrm{I}_{\mathrm{G}}$ ratio is indicative of (a) more structural defects (b) the increase of the amount of amorphous carbon (c) the decrease of crystalline size $[33,34]$. The result also indicates that T3-RGO displayed enhanced structural disorder, which can be ascribed to the higher defect density of in-plane graphitic crystalline $\mathrm{sp}^{2}$ domains generated by reduction and functionalization [33]. Additionally, T3-RGO showed higher intensity of 2D mode band at $2691 \mathrm{~cm}^{-1}$, revealing the formation of few-layered T3-RGO sheets [35-36]. The layer number of 
T3-RGO sheets was smaller than that of T2-RGO sheets, which is favorable for the uniform dispersion of T3-RGO in the polymer matrix.
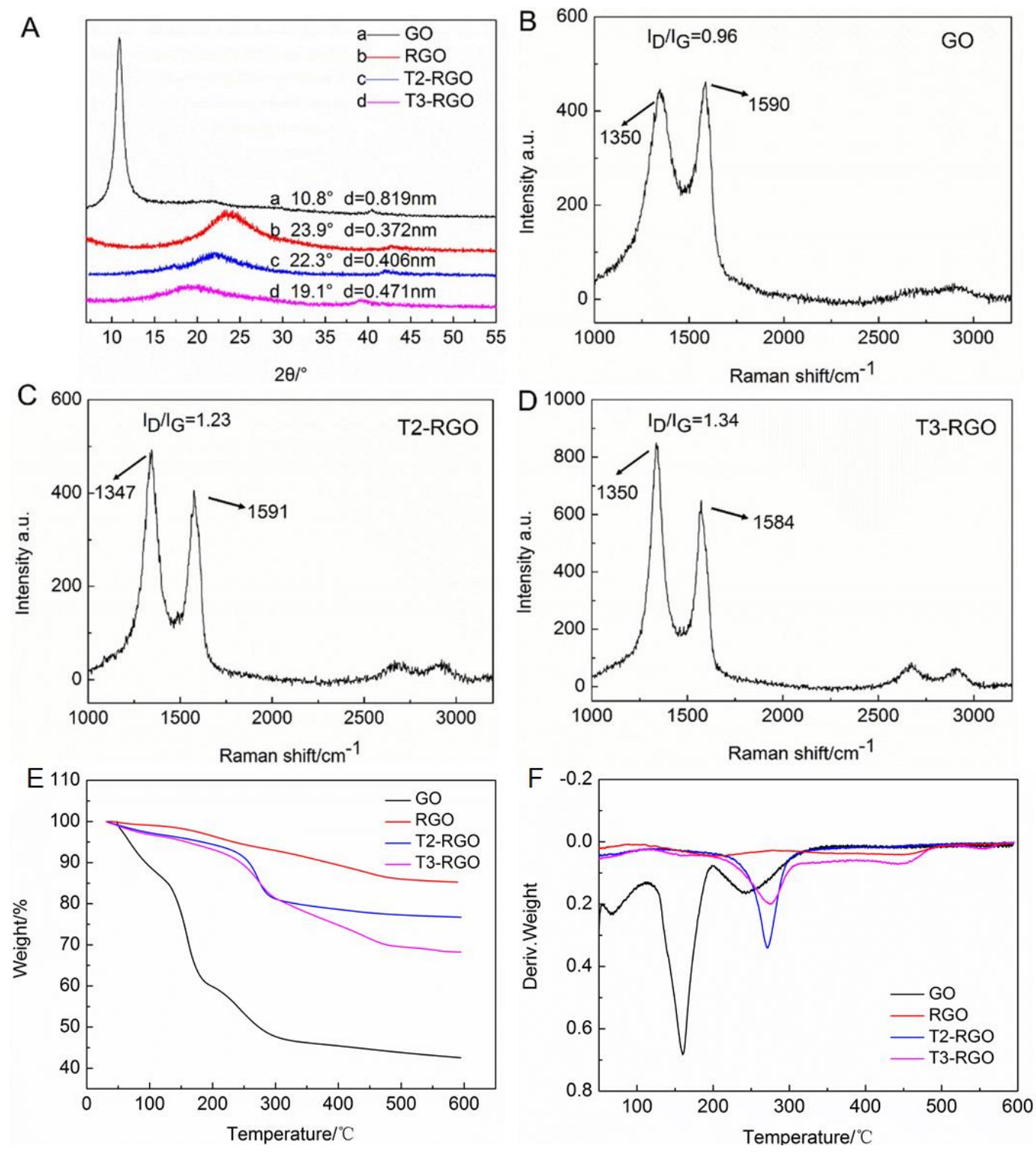

Figure 4. (A) XRD patterns of GO, RGO, T2-RGO and T3-RGO; Raman spectrum of GO (B),

T2-RGO (C) and of T3-RGO (D); TG (E) and DTG (F) curves of GO, RGO, T2-RGO and T3-RGO.

Figures $4 \mathrm{E}$ and $4 \mathrm{~F}$ shows $\mathrm{TG}$ and DTG curves of GO, RGO, T2-RGO and T3-RGO nanosheets. GO was thermally unstable and the weight loss process started below $100{ }^{\circ} \mathrm{C}$ and the major weight loss occurred in the range from $100{ }^{\circ} \mathrm{C}$ to $300{ }^{\circ} \mathrm{C}$, 
owing to the pyrolysis of oxygen-containing functional groups [22]. After chemical reduction, the thermal stability of RGO was greatly increased, and it displayed a weight loss of only $14.8 \%$, much lower than that $57.4 \%$ for GO. With the incorporation of $\mathrm{T} 2$ and $\mathrm{T} 3$, new degradation peaks were observed over the region of 200-310 ${ }^{\circ} \mathrm{C}$, which can be assigned to the loss of titanate coupling agent. The weight loss of T2-RGO and T3-RGO were $23.28 \%$ and $31.76 \%$, respectively; and the difference was directly related to the graft efficiency of coupling agents. The higher weight loss of T3-RGO suggests that the graft efficiency of T3 with higher branching degree was higher than that of $\mathrm{T} 2$.

3.2. In situ Polymerization of RGO Functionalized Waterborne Poly(urethane-acrylate) Nanoemuslions and Stability Characterization.

T2-RGO or T3-RGO were introduced into the reaction system with monomers during the preparation process of polyurethane (PU) prepolymer (Stage 1 and Stage 2), followed by the phase inversion process (Stage 3) and then radical polymerization of vinyl-terminated polyurethane prepolymer with vinyl monomers (Stage 4), as illustrated in the schematic model (Figure 5). During the polymerization, T2-RGO and T3-RGO were able to react with isophorone diisocyanate and became a segment of polyurethane chains instead of just forming chemical bonds inbetween RGO nanosheets and polyurethane chain; which can effectively improve the compatibility between RGO nanosheets and polymer chains, and further promote the nanoscopic dispersion of RGO in WPUA. 

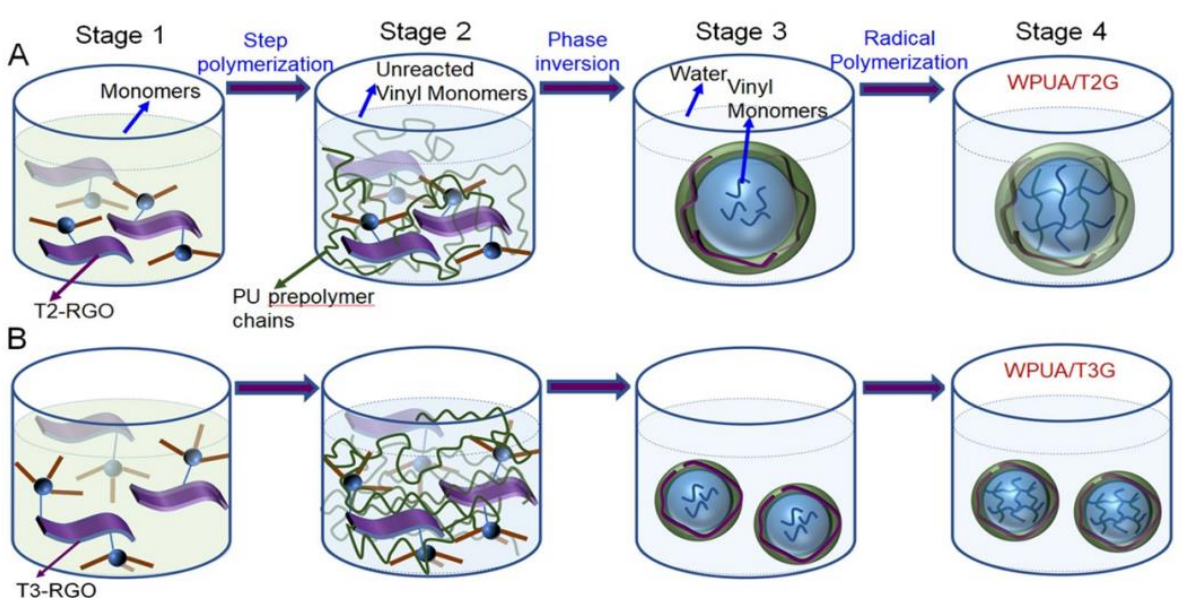

hains
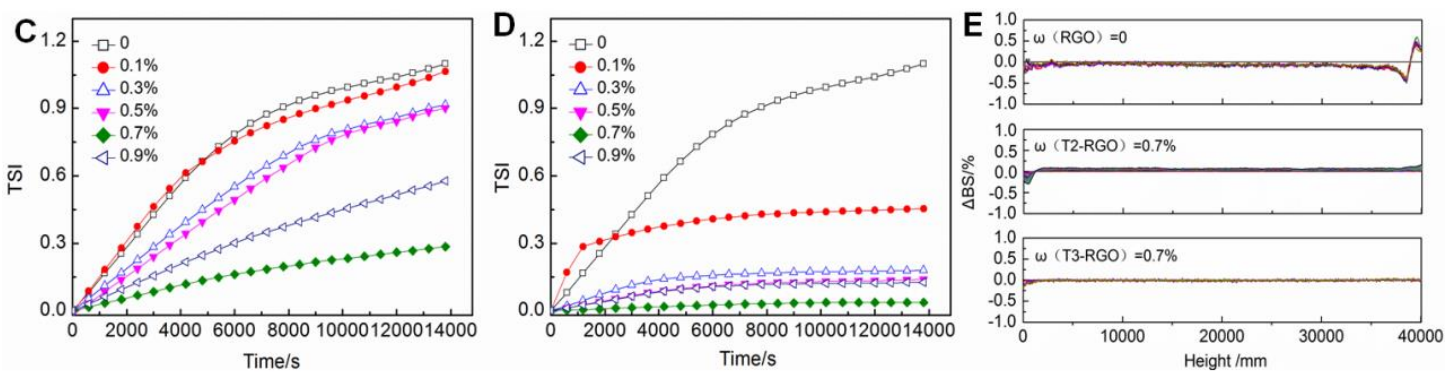

Figure 5. Schematic illustration of preparing WPUA/T2G nanoemulsion (A) and WPUA/T3G nanoemulsion (B); Turbiscan stability index (TSI) values of WPUA/T2G nanoemulsions (C) and WPUA/T3G nanoemulsions (D); (E) The $\Delta$-Backscattering $(\Delta \mathrm{BS})$ profiles of WPUA/T2G and WPUA/T3G nanoemulsions.

The stability of WPUA/T2G and WPUA/T3G nanoemulsions was estimated by turbiscan stabilizer, which is an effective approach to detect the destabilization phenomena. Turbiscan stability index (TSI) was calculated to monitor the coalescence kinetics versus aging time. The lower the TSI value is, the better the emulsion stability is. The TSI values and the increasing rate of TSI for WPUA/T3G nanoemulsions were lower than that of WPUA/T2G nanoemulsions (Figure 5C, 5D). This proves that $\mathrm{T} 2-\mathrm{RGO}$ and $\mathrm{T} 3-\mathrm{RGO}$ nanosheets can be functionalized as stabilizing agents to improve the nanoemulsion stability, especially for T3-RGO nanosheets of higher branching degree. $\triangle \mathrm{BS}$ profiles of WPUA/T2G and WPUA/T3G 
nanoemulsions were also obtained, as shown in Figure 5E. The left, middle and right domains of the $\triangle \mathrm{BS}$ profiles represented the bottom, middle and top part of the samples, respectively. The variation of backscattering light intensity in the middle domain was related to the change of particle size (renion or flocculation). The changes in the left and right domains represented the migration of colloidal particles (precipitation or floatation). Only minimal variation was observed for WPUA/T3G nanoemulsion when the content of T3-RGO was $0.7 \%$, indicative of the enhanced stability of WPUA/T3G nanoemulsion.

With the increased content of RGO from $0.0 \%$ to $0.7 \%$, the average particle size of WPUA/T2G increased from $21.19 \mathrm{~nm}$ to $131.9 \mathrm{~nm}$, and from $21.19 \mathrm{~nm}$ to 54.65 nm for WPUA/T3G (Figure 6A, 6B). WPUA/T3G showed much smaller average particle size compared to WPUA/T2G, indicating the incorporation of T3-RGO was more effective in enhancing the compatibility and interactions between RGO and polymer chains by forming more compact colloidal particles with decreased article size (Figure 6B). TEM results also showed a similar trend (Figure 6C, 6D). Both WPUA/T2G and WPUA/T3G colloidal particles displayed core-shell morphology, and the core-shell periphery became more remarkable with the increased content of RGO. Notably, the particle size dramatically increased to $210.0 \mathrm{~nm}$ and $108.3 \mathrm{~nm}$ for WPUA/T2G and WPUA/T3G, and a semi-enclosed core-shell structure was detected when the T3-RGO content reached $0.9 \%$. It may be difficult for the polymer to encapsulate RGO nanosheets inside the colloid particles at high contents of RGO, although it is a 2D material of high flexibility. It is also worthy to note that the particle 
size of WPUA/T2G is much smaller than the lateral size of RGO. The main reason is that deformation, edge curling, or RGO nanosheet breakdown took place in RGO nanosheets with external stress (such as vigorous mechanical stirring utilized in this manuscript) when it is encapsulated by polymer chains, especially when chemical bonds are built between RGO and polymer; Moreover, graphene nanosheet shows pronounced flexibility even at higher thickness [38]
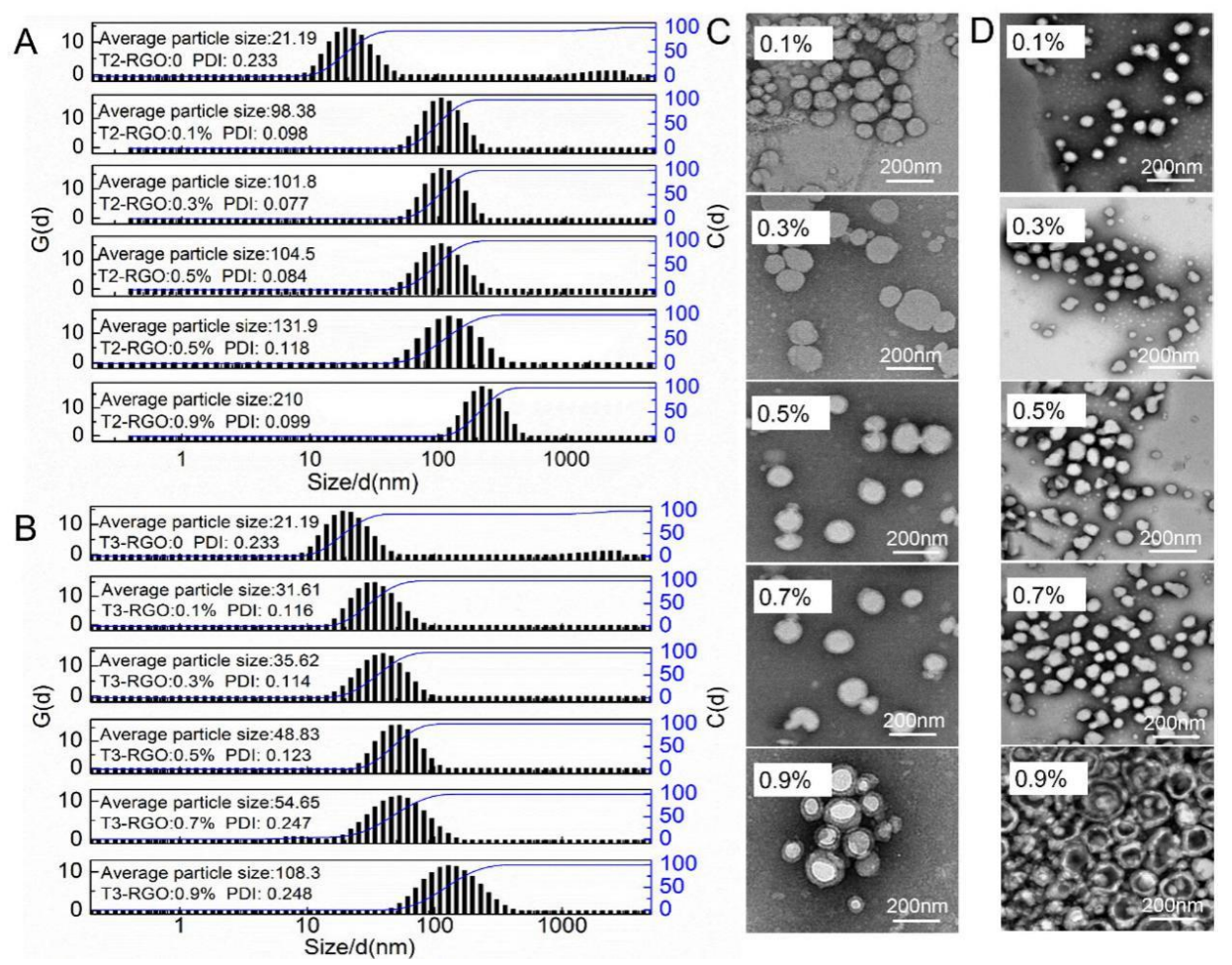

Figure 6. Particle size and distribution of T2-RGO functionalized waterborne (polyurethane-acrylate) nanoemulsions (WPUA/T2G) (A) and T3-RGO functionalized waterborne (polyurethane-acrylate) nanoemulsions (WPUA/T3G) (B) with different content of T2-RGO or T3-RGO; TEM images of WPUA/T2G colloidal particles (C) and WPUA/T3G colloidal particles (D).

3.3. Water Resistance, Mechanical Properties and Morphology of Nanocomposite Films. 
Figures 7A and 7B display the water absorption and contact angle for WPUA/T2G and WPUA/T3G nanocomposite films with different contents of RGO. The water absorption decreased first and then increased with the increasing RGO content; while the contact angle increased first and then decreased. These phenomena suggest that the barrier properties of WPUA nanocomposite film were enhanced with the incorporation of RGO nanosheets. Compared with WPUA/T2G nanocomposite film, WPUA/T3G nanocomposite film displayed higher water resistance. The mechanism behind the performance may be explained as follows: denser WPUA/T3G nanocomposite films can be formed owing to its smaller particle size; enhanced chemical bonding and hydrogen bonding can be generated between T3-RGO graphene nanosheets and polymer chains, leading to the formation of more uniform organic-inorganic networks, which is favorable for increasing the water resistance. However, when the RGO content increased to $0.9 \%$, the performance decreased, which may be attributed to the weakened phase compatibility and increased phase aggregation.

Figures 7C and 7D showed the stress-strain curves of WPUA/T2G and WPUA/T3G nanocomposite films. With incorporation of $0.7 \% \mathrm{~T} 2-\mathrm{RGO}$, the tensile strength increased from $17.78 \mathrm{MPa}$ to $32.01 \mathrm{MPa}$, and the elongation at break increased from $249 \%$ to $366 \%$. In contrast, the tensile strength and elongation at break of WPUA/T3G with $0.7 \%$ T3-RGO were $40.01 \mathrm{MPa}$ and $424 \%$, respectively. It can be concluded that the incorporation of RGO nanosheets greatly improved the interactions between RGO nanosheets and polymer chains, increased the film compactness, and 
thereby increased the mechanical properties.
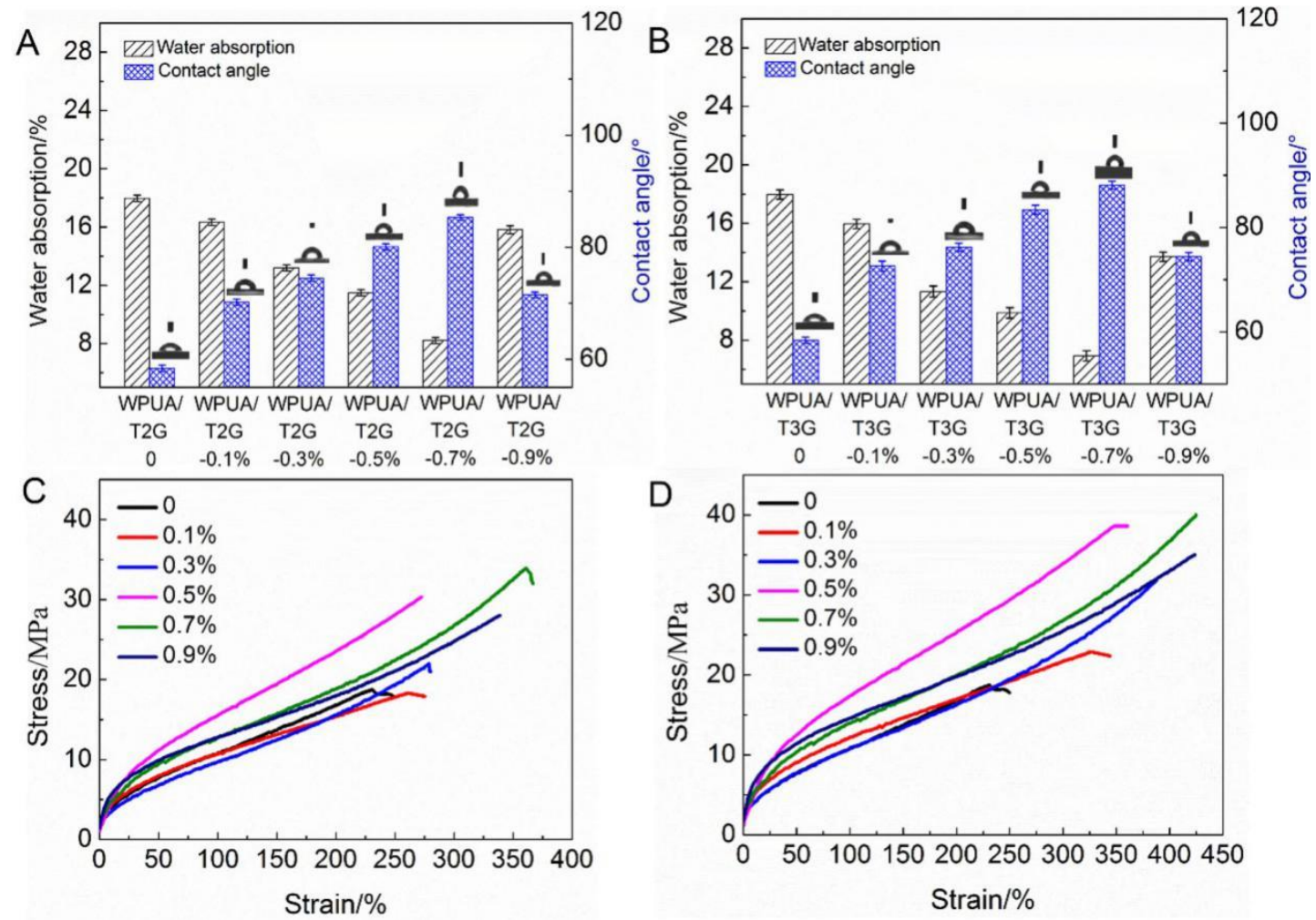

Figure 7. (A) Water adsorption of WPUA/T2G films with different T2-RGO content; (B) Water adsorption of WPUA/T3G films with different T3-RGO content; (C) Tensile strength of WPUA/T2G films with different T2-RGO content; (D) Tensile strength of WPUA/T3G films with different T3-RGO content.

SEM surface and cross-section morphology of WPUA, WPUA-T2G and WPUA-T3G nanocomposite films was also provided, as shown in Figure.8. Pristine WPUA (Figure.8A) exhibits smooth surface morphology, and the fracture surface also displays relative smooth morphology with propagated crack lines (Figure.8D). With the incorporation of T2-RGO and T3-RGO, the roughness and tortuous paths increase. It can be explained by the good interactions between RGO nanosheets and WPUA matrix [24]. In comparison with WPUA-T2G film (Figure. 8B), WPUA-T3G film displays more prominent roughness (Figure. 8C), and the roughness continues 
homogeneously throughout the nanocomposite film. This phenomenon excludes the possibility of pinhole defects in the nanocomposite film, which is favorable to inhibit the penetration of corrosive medium into film [39]. The size of micro-cracks for the fracture surface of WPUA-T3G decreased (Figure. 8E and Figure. 8F) due to the increased number of RGO nanosheets per unit volumes, which was generated by the better dispersion of RGO in nanocomposites. It is reported that the micro-cracking can release the crack tip stresses and dissipate additional amount of energy. As a consequence, WPUA-T3G nanocomposite film exhibits improved mechanical properties, as demonstrated by the above results from tensile strength.
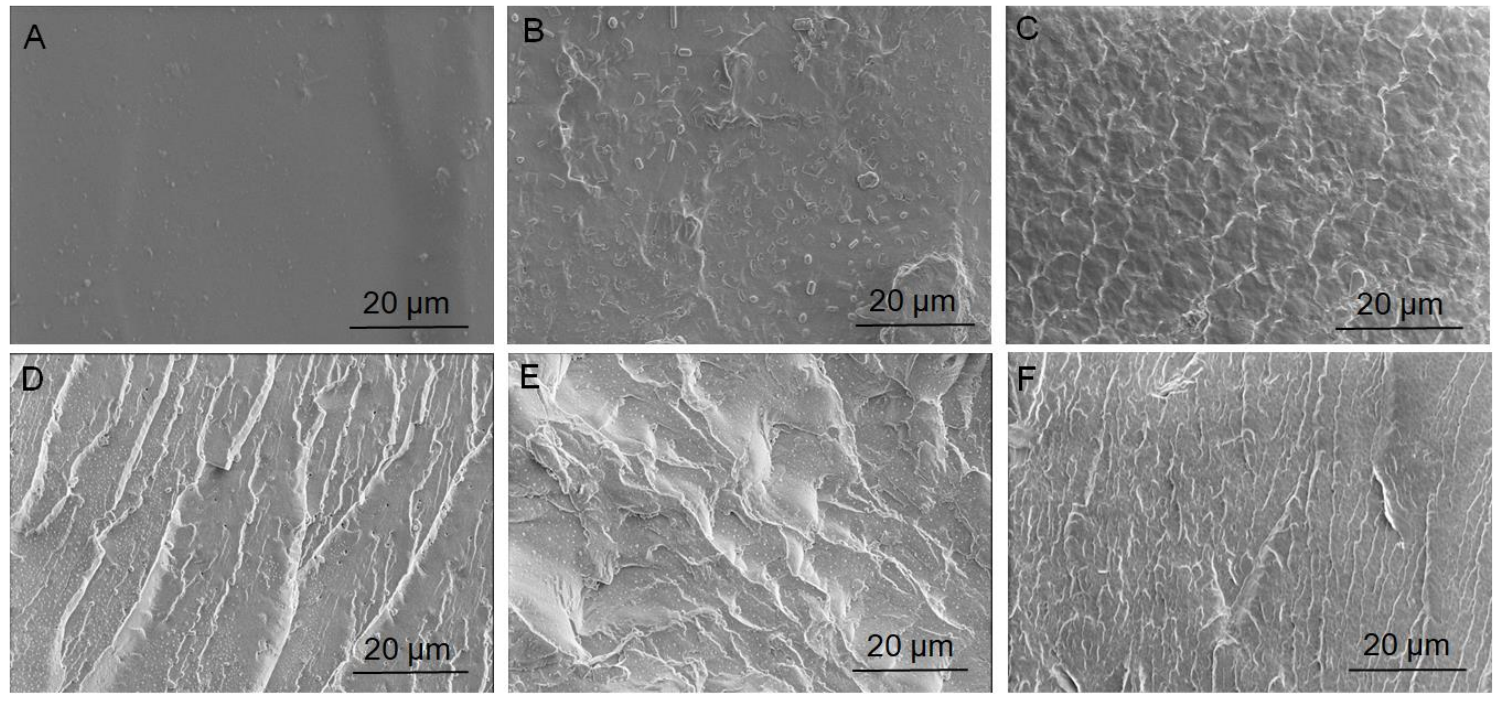

Figure 8. SEM surface (upper) and cross-section (bottom) morphology of (A,D) WPUA, (B,E) WPUA-T2G and (C,F) WPUA-T3G nanocomposite films

\subsection{Corrosion Resistance Analysis of Nanocomposite Coatings.}

EIS was utilized to evaluate the anticorrosive properties of WPUA/T2G and WPUA/T3G nanocomposite coatings, and the Bode and Nyquist plots are shown in

Figure 9. In general, the impedance modulus $|\mathrm{Z}|$ at the lowest frequency in Bode 
impedance plots (Figure 9A, B) can be used as a semi-quantitative indicator of corrosion resistance. The impedance modulus of WPUA coating was only $1.12 \times 10^{5}$ $\Omega . \mathrm{cm}^{2}$, and increased to $6.93 \times 10^{6} \Omega . \mathrm{cm}^{2}$ with the incorporation of only $0.7 \%$ T2-RGO.

The impedance modulus of WPUA/T3G was significantly increased and reached $1.15 \times 10^{8} \Omega . \mathrm{cm}^{2}$ with $0.7 \% \mathrm{~T} 3-\mathrm{RGO}$, indicating enhanced corrosion resistance.
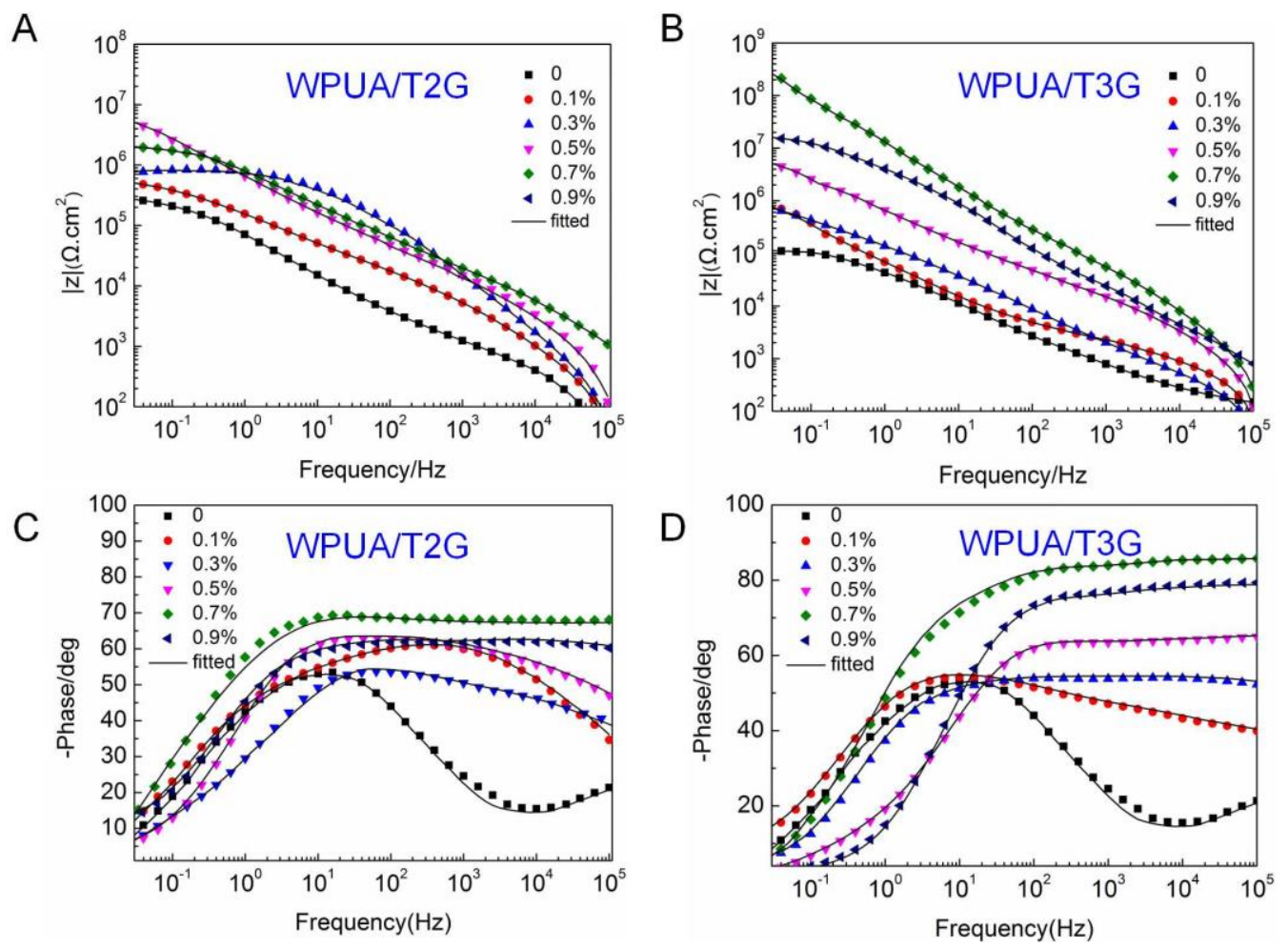

Figure 9. Bode impedance plots for WPUA/T2G (A) and WPUA/T3G (B) nanocomposite coatings prepared with different content of graphene before salt spray; Bode phase plots for WPUA/T2G (C) and WPUA/T3G (D) nanocomposite coatings prepared with different content of graphene before salt spray.

Bode phase angles for WPUA, WPUA/T2G and WPUA/T3G nanocomposite coatings are also presented (Figure 9C, 9D). Generally, the corrosion process can be divided into two stages. At the first stage, corrosive electrolytes penetrated into the 
coating through the inherent micro-pores, resulting in decreased coating resistance $\mathrm{R}_{\mathrm{c}}$ [37]. At the second stage, the electrolyte reached the metal interface, leading to metal corrosion, two-time constant was thereby generated. WPUA exhibited two-time constant, indicating poor anticorrosion properties. In contrast, WPUA/T2G and WPUA/T3G nanocomposite coatings only displayed one-time constant. Moreover, the phase angle for WPUA/T3G-0.7\% nanocomposite coating was increased to $83.9^{\circ}$. The higher phase angle at high frequency of $10^{5} \mathrm{~Hz}$ corresponds to the enhanced barrier properties of coatings [22].

Table 1. Electrochemical parameters obtained from impedance data of WPUA/T2G and WPUA/T3G nanocomposite coatings prepared with different contents of graphene before salt spray; The values are the mean of three replicates and $( \pm)$ corresponds to the standard deviations.

\begin{tabular}{|c|c|c|c|c|c|c|}
\hline \multirow[b]{2}{*}{ Sample } & \multicolumn{3}{|c|}{$\mathrm{CPE}_{\text {coat }} \times 10^{8} /$} & \multicolumn{3}{|c|}{$\mathrm{CPE}_{\mathrm{dl}} \times 10^{6} /$} \\
\hline & $\operatorname{R}_{\text {coat }}\left(\Omega . \mathrm{cm}^{2}\right)$ & $\gamma_{0}\left(\Omega^{-1} \mathrm{~cm}^{-2} \mathrm{~s}^{\mathrm{n}}\right)$ & $\mathrm{n}$ & $\operatorname{R}_{\mathrm{CT}}\left(\Omega . \mathrm{cm}^{2}\right)$ & $\begin{array}{c}\gamma_{0} \\
\left(\Omega^{-1} \mathrm{~cm}^{-2} \mathrm{~s}^{\mathrm{n}}\right)\end{array}$ & $\mathrm{n}$ \\
\hline Pure WPUA & $8.41 \times 10^{4} \pm 81$ & $11.5 \pm 2.77$ & $0.78 \pm 0.03$ & $1.20 \times 10^{5} \pm 77$ & $38.3 \pm 3.35$ & $0.51 \pm 0.02$ \\
\hline WPUA/T2G-0.1\% & $4.36 \times 10^{5} \pm 113$ & $464 \pm 50.2$ & $0.62 \pm 0.02$ & -- & -- & -- \\
\hline WPUA/T2G-0.3\% & $6.31 \times 10^{5} \pm 107$ & $517 \pm 41.7$ & $0.69 \pm 0.02$ & -- & -- & -- \\
\hline WPUA/T2G-0.5\% & $7.64 \times 10^{5} \pm 91$ & $30.9 \pm 3.21$ & $0.81 \pm 0.01$ & -- & -- & -- \\
\hline WPUA/T2G-0.7\% & $7.31 \times 10^{6} \pm 98$ & $68.3 \pm 2.69$ & $0.86 \pm 0.02$ & -- & -- & -- \\
\hline WPUA/T2G-0.9\% & $8.05 \times 10^{5} \pm 112$ & $32.6 \pm 4.72$ & $0.73 \pm 0.01$ & -- & -- & -- \\
\hline WPUA/T3G-0.1\% & $9.12 \times 10^{5} \pm 96$ & $217 \pm 12.3$ & $0.62 \pm 0.01$ & -- & -- & -- \\
\hline WPUA/T3G-0.3\% & $1.01 \times 10^{6} \pm 87$ & $378 \pm 34.1$ & $0.63 \pm 0.02$ & -- & -- & -- \\
\hline WPUA/T3G-0.5\% & $6.92 \times 10^{6} \pm 102$ & $42.0 \pm 2.21$ & $0.69 \pm 0.01$ & -- & -- & -- \\
\hline WPUA/T3G-0.7\% & $1.39 \times 10^{8} \pm 94$ & $0.358 \pm 0.154$ & $0.93 \pm 0.01$ & -- & -- & -- \\
\hline WPUA/T3G-0.9\% & $1.98 \times 10^{7} \pm 86$ & $1.84 \pm 0.271$ & $0.80 \pm 0.02$ & -- & -- & -- \\
\hline
\end{tabular}

The equivalent electrical circuits (EEC) presented in Figure 10A and 10B were utilized to simulate the impedance data. In the EEC, $R_{s}$ is the solution resistance, $\mathrm{CPE}_{\text {coat }}$ and $\mathrm{CPE}_{\mathrm{dl}}$ represents non-ideal capacitance of coating and non-ideal capacitance of double layer. $\mathbf{R}_{\text {coat }}$ is coating resistance, which is considered as an indicator to evaluate the barrier property of a coating by which it impedes the 
penetration of corrosive medium into the coating [21]. $\mathrm{R}_{\mathrm{CT}}$ represents the charge transfer resistance, i.e. the resistance generated by the interface of nanocomposite coating and electrolyte interface. The derived electrochemical parameters for WPUA, WPUA/T2G and WPUA/T3G are listed in Table 1. Model A was utilized when the electrolyte has not arrived at the metal substrate, model B was selected when the corrosion reached the coating/metal interface. Model B is only utilized for simulating the impedance data of WPUA, since WPUA displays two-time constant from its Bode phase plots. $\mathrm{R}_{\text {coat }}$ of WPUA, WPUA/T2G-0.7\% and WPUA/T3G-0.7\% was $8.41 \times 10^{4}$ $\Omega . \mathrm{cm}^{2}, 7.31 \times 10^{6} \Omega . \mathrm{cm}^{2}$ and $1.39 \times 10^{8} \Omega . \mathrm{cm}^{2}$, respectively. The variation of $\mathrm{R}_{\text {coat }}$ with T2-RGO and T3-RGO content also showed a similar trend as the impedance modulus. Higher $\mathrm{R}_{\text {coat }}$ can be explained by the good dispersibility and barrier properties of functionalized graphene in the nanocomposite coating, i.e. WPUA/T3G-0.7\% displays the most prominent barrier properties compared with WPUA and WPUA/T2G-0.7\% [37]. Significant increase in the capacitive arcs was also observed for the WPUA/T3G nanocomposite coatings (Figure 10C, D). This further demonstrates that the corrosion resistance can be enhanced by in-situ incorporating functionalized RGO during the preparation process of polyurethane prepolymer, especially for RGO functionalized with dendritic titanium coupling agent containing three branched dioctylpyrophosphate. 

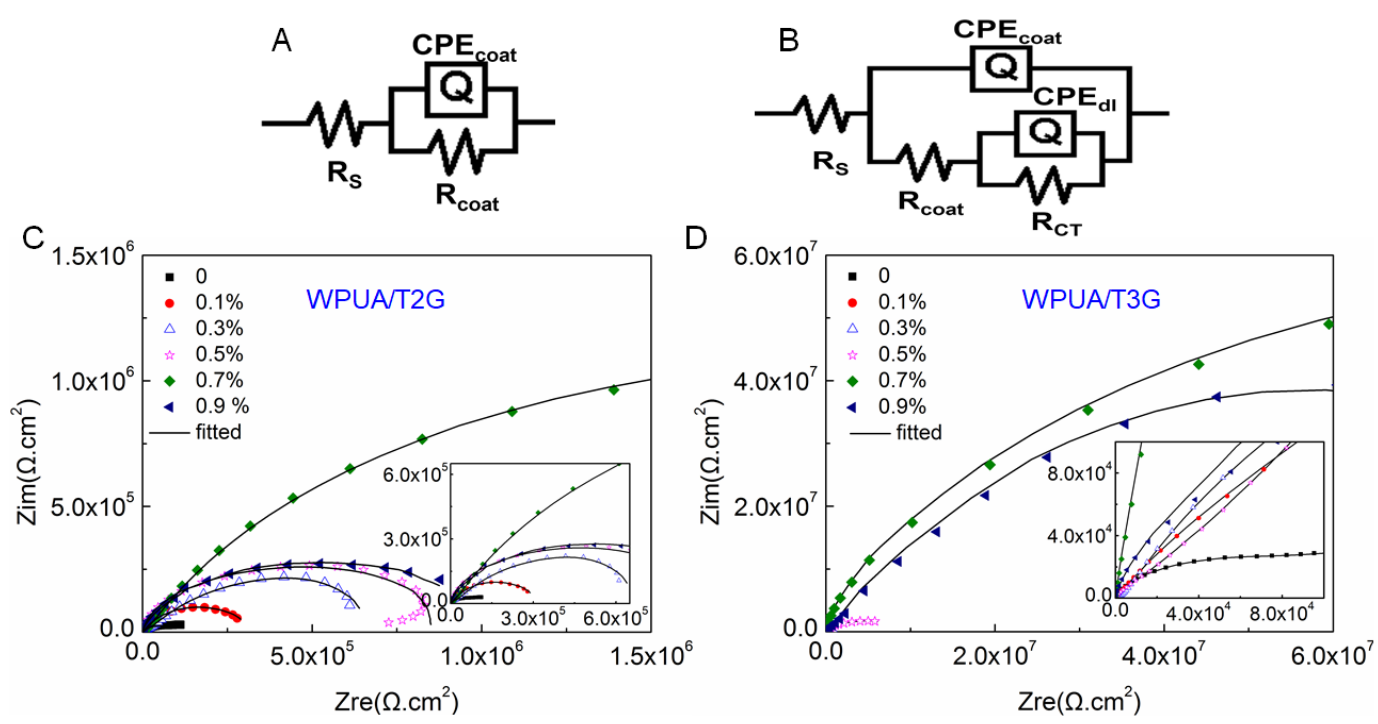

Figure 10. Electrical equivalent circuit models (A, B) for nanocomposite coatings; Nyquist plots of WPUA/T2G (C) and WPUA/T3G (D) nanocomposite coatings with different graphene content before salt spray test.

The anticorrosion properties of WPUA/T2G-0.7\% and WPUA/T3G-0.7\% nanocomposite coatings at different exposure times are shown in Figure 11, and the related electrochemical parameters are listed in Table 2. The impedance modulus of WPUA/T2G-0.7\% was decreased to $1.36 \times 10^{4} \Omega . \mathrm{cm}^{2}$ after exposure for $480 \mathrm{~h}, \mathrm{R}_{\text {coat }}$ decreased from $7.31 \times 10^{6} \Omega . \mathrm{cm}^{2}$ to $7.51 \times 10^{3} \Omega . \mathrm{cm}^{2}$, and $\mathrm{CPE}_{\text {coat }}$ increased by two orders. Two-time constant was also detected for WPUA/T2G nanocomposite coatings after $120 \mathrm{~h}$ exposure, which may be due to the coating defects generated by the inhomogeneous distribution of T2-RGO or graphene aggregation. It is also found that $\mathrm{R}_{\mathrm{CT}}$ decreased by one order, which is attributed to the coating delamination progress from defect size [40]. The impedance modulus of WPUA/T3G-0.7\% decreased from $1.15 \times 10^{8}$ to $1.99 \times 10^{6} \Omega . \mathrm{cm}^{2}$, and $\mathrm{R}_{\text {coat }}$ decreased from $1.39 \times 10^{8} \Omega . \mathrm{cm}^{2}$ to $1.54 \times 10^{6}$ $\Omega . \mathrm{cm}^{2}$ after $480 \mathrm{~h}$ immersion. The higher $\mathrm{R}_{\text {coat }}$ for WPUA/T3G-0.7\% and decreased 
$\mathrm{CPE}_{\text {coat }}$ at all the applied exposure times demonstrate an enhanced long-term corrosion resistance. Li et al. physically blended graphene functionalized with titanate coupling agent TM-200S with waterborne polyurethane and fabricate composite coatings, and blisters were observed on the coating after $48 \mathrm{~h}$ exposure to $\mathrm{NaCl}$ solution [14]. In contrast, WPUA/T3G-0.7\% nanocomposite coating displayed greatly enhanced long-term corrosion resistance.
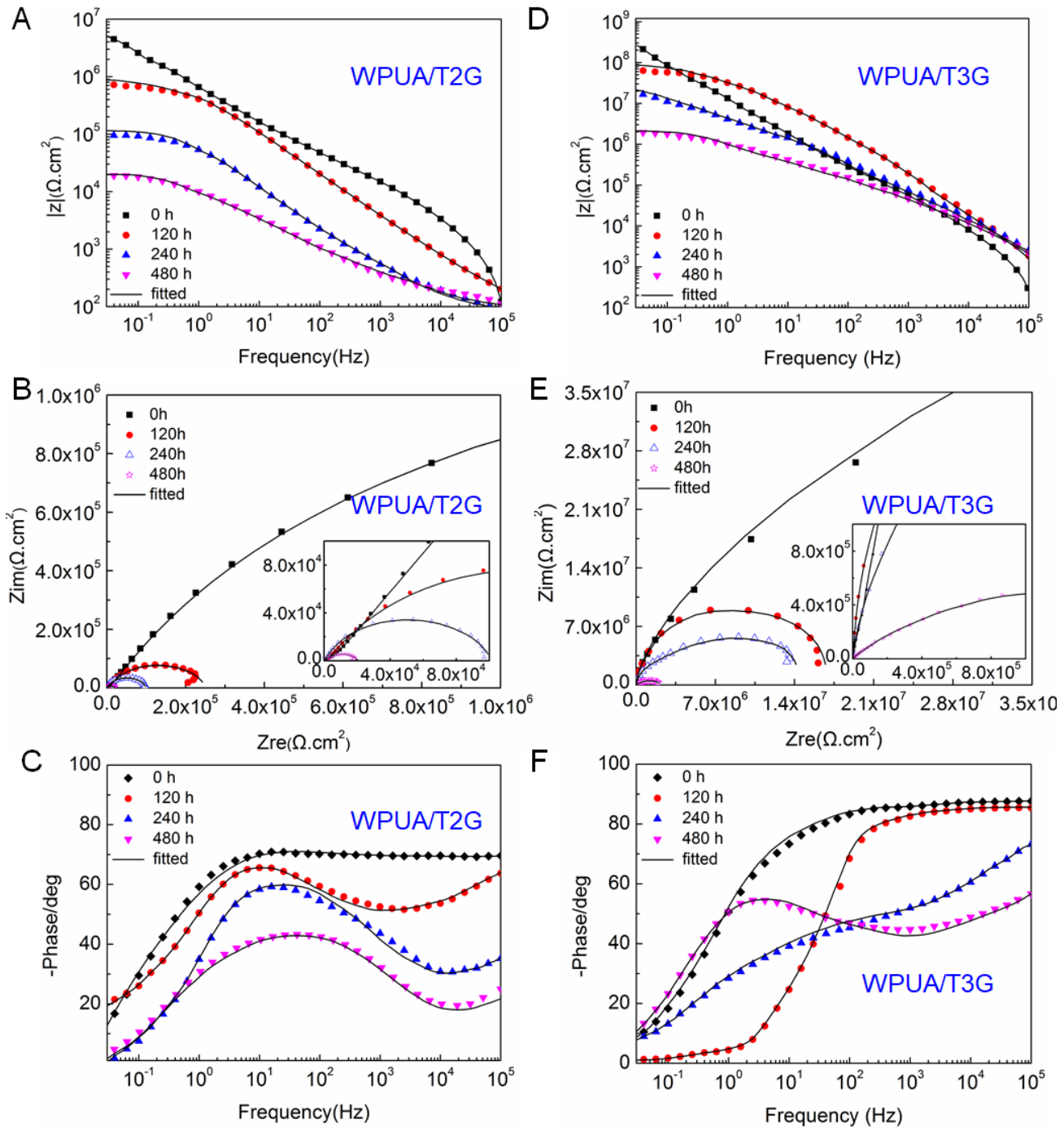

Figure 11. Bode impedance plots (A), Nyquist plots (B) and Bode phase plots (C) of WPUA/T2G nanocomposite coating at different exposure times. Bode impedance plots (D), Nyquist plots (E) and Bode phase plots (F) of WPUA/T3G nanocomposite coating at different exposure times. 
Table 2. Electrochemical parameters obtained from impedance data of WPUA/T2G and WPUA/T3G nanocomposite coatings at different exposure times; The values are the mean of three replicates and $( \pm)$ corresponds to the standard deviations.

\begin{tabular}{llllrrr}
\hline \multicolumn{1}{c}{ Sample } & $\mathrm{R}_{\text {coat }}\left(\Omega . \mathrm{cm}^{2}\right)$ & $\begin{array}{l}\mathrm{CPE} \text { coat } \times 10^{8} / \\
\gamma_{0}\left(\Omega^{-1} \mathrm{~cm}^{-2} \mathrm{~s}^{\mathrm{n}}\right)\end{array}$ & $\mathrm{n}$ & $\mathrm{R}_{\mathrm{CT}}\left(\Omega . \mathrm{cm}^{2}\right)$ & $\begin{array}{c}\mathrm{CPE} \mathrm{dl} \times 10 / \\
\gamma_{0}\left(\Omega^{-1} \mathrm{~cm}^{-2} \mathrm{~s}^{\mathrm{n}}\right)\end{array}$ & $\mathrm{n}$ \\
\hline WPUA/T2G-0.7\%-0h & $7.31 \times 10^{6} \pm 98$ & $68.3 \pm 2.69$ & $0.86 \pm 0.02$ & -- & -- & - \\
WPUA/T2G-0.7\%-120h & $1.92 \times 10^{5} \pm 101$ & $87.8 \pm 2.16$ & $0.69 \pm 0.02$ & $6.53 \times 10^{5} \pm 90$ & $18.1 \pm 1.75$ & $0.67 \pm 0.02$ \\
WPUA/T2G-0.7\%-240h & $8.42 \times 10^{4} \pm 92$ & $559 \pm 14.1$ & $0.80 \pm 0.01$ & $1.02 \times 10^{5} \pm 83$ & $424 \pm 30.5$ & $0.74 \pm 0.01$ \\
WPUA/T2G-0.7\%-480h & $7.51 \times 10^{3} \pm 73$ & $3190 \pm 133$ & $0.89 \pm 0.03$ & $2.10 \times 10^{4} \pm 79$ & $2840 \pm 262$ & $0.59 \pm 0.03$ \\
WPUA/T3G-0.7\%-0h & $1.39 \times 10^{8} \pm 94$ & $0.358 \pm 0.154$ & $0.93 \pm 0.01$ & -- & -- & -- \\
WPUA/T3G-0.7\%-120h & $5.69 \times 10^{7} \pm 103$ & $3.72 \pm 0.181$ & $0.80 \pm 0.02$ & -- & -- & -- \\
WPUA/T3G-0.7\%-240h & $1.01 \times 10^{7} \pm 91$ & $15.2 \pm 1.24$ & $0.75 \pm 0.01$ & -- & -- & - \\
WPUA/T3G-0.7\%-480h & $1.54 \times 10^{6} \pm 93$ & $71.5 \pm 1.22$ & $0.86 \pm 0.02$ & $9.16 \times 10^{5} \pm 72$ & $98.3 \pm 3.51$ & $0.71 \pm 0.01$ \\
\hline
\end{tabular}

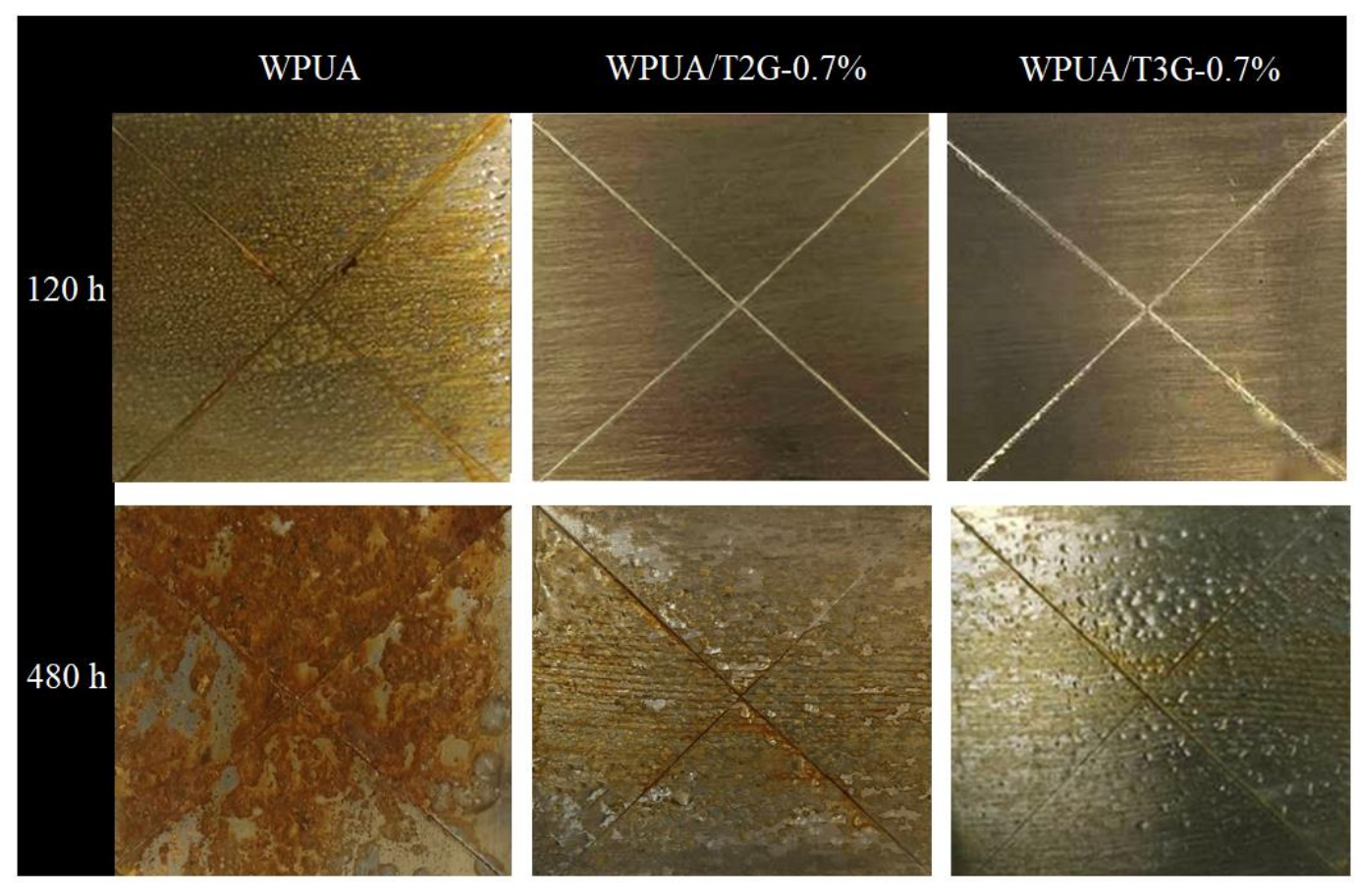

Figure 12. Images of coated tinplates subjected to the salt spray test at different exposure times.

No blister, rust, pitting and corrosion diffusion were observed for WPUA/T2G-0.7\% and WPUA/T3G-0.7\% coated tinplates after $120 \mathrm{~h}$ exposure (Figure 12); in sharp contrast to the severe corrosion diffusion detected for WPUA coated tinplate. Rust and pitting corrosion along the cutting part were detected after exposure for $480 \mathrm{~h}$. The corrosion damage of WPUA/T2G- $0.7 \%$ coated tinplate was 
much worse than that of tinplate with WPUA/T3G-0.7\% coating, demonstrating the increased salt spray resistance of WPUA/T3G-0.7\%.

\section{Conclusion}

Functionalizing RGO with titanate coupling agents have been demonstrated to be an effective approach to prepare large-size, few-layed RGO nanosheets affording improved dispersion stability in polymer matrix. The titanate coupling agent acts as a "bridge" to covalently connect RGO and WPUA, and consequently increase the compatibility and interactions between RGO and polymer chains to form waterborne functionalized graphene/poly (urethane-acrylate) nanocomposite coatings of high performance. The water resistance, tensile strength and elongation at break of nanocomposite films are significantly improved and reach the maximum when the content of graphene is $0.7 \%$. Compared with WPUA, the tensile strength, elongation at break, impedance modulus and coating resistance all have been greatly improved. The WPUA/T3G nanocomposite coating demonstrates a superior performance to that of WPUA/T2G, and better than the reported performance of solventborne polyurethane/graphene composite coating as well. These results indicate that WPUA/T3G is a promising material for application in anticorrosion coatings to replace the commercial solventborne coatings. The developed fabrication approach in this work can be easily expanded to produce anticorrosion coatings with other waterborne polymer matrixes.

\section{- Author information}




\section{Corresponding Author}

*E-mail:whh@sust.edu.cn, wseaflower@126.com

*E-mail: feiguiqiang@sust.edu.cn

\section{- Acknowledgements}

The authors express sincere thanks to the Key Research and Development Program of Shaanxi Province--International Cooperation Project (No. 2018KW-007), Key Research and Development Program of Shaanxi Province (No. 2017GY154), Innovation Supporting Plan of Shaanxi Province-Innovation Research Team (No. 2018TD-015), National Natural Science Foundation of China (No. 51373091), Scientific Research Foundation (SRF) for Returned Overseas Chinese scholars (ROCS), State Education Ministry (SEM) (No. [2012]1707).

\section{- References}

[1] N. Parhizkar, T. Shahrabi, B. Ramezanzadeh, Steel surface pre-treated by an advance and eco-friendly cerium oxide nanofilm modified by graphene oxide nanosheets; electrochemical and adhension measurements, J. Alloy. Compd. 747 (2018)109-123.

[2] B. Ramezanzadeh, B. Karimi, M. Ramezanzadeh, M. Rostami, Synthesis and characterization of polyaniline tailored graphene oxide quantum dot as an advance and highly crystalline carbon-based on luminescent nanomaterial for fabrication of an effective anti-corrosion epoxy system on mild steel, J. Taiwan. Inst. Chem. E. 000 (2018) 1-14. 
[3] P. Haghdadeh, M. Ghaffari, B. Ramezanzadeh, G. Bahlakeh, M. R. Saeb, The role of functionalized graphene oxide on the mechanical and anti-corrosion properties of polyurethane coating, J. Taiwan. Inst. Chem. E. 86 (2018) 199-212.

[4] T. Wan, D. Chen, Synthesis and properties of self-healing waterborne polyurethanes containing disulfide bonds in the main chain, J. Mater. Sci. 52 (2017) 197-207.

[5] G. Christopher, M. A. Kulandainathan, G. Harichandran, Biopolymers nanocomposite for material protection: Enhancement of corrosion protection using waterborne polyurethane nanocomposite coatings, Prog. Org. Coat. 99 (2016) 91-102.

[6] M. A. Deyab, A. A. Nada, A. Hamdy, Comparative study on the corrosion and mechanical properties of nano-composite coatings incorporated with $\mathrm{TiO}_{2}$, nano-particles, $\mathrm{TiO}_{2}$, nano-tubes, and $\mathrm{ZnO}$ nano-flowers, Prog. Org. Coat. 105 (2017) 245-251.

[7] M. A. EI-Fattah, A. M. Hasan, A. M. Keshawy, A. M. EI Saeed, O. M. Aboelenien, Nanocrystalline cellulose as an eco-friendly reinforcing additive to polyurethane coating for augmented anticorrosive behavior, Carbohyd. Polym. 183 (2018) 311-318.

[8] J. Li, L. Gan, Y. Liu, S. Mateti, W. Lei, Y. Chen, J. Yang, Boron nitride nanosheets reinforced waterborne polyurethane coatings improving corrosion resistance and antifriction properties, Euro. Polym. J. 104 (2018) 57-63.

[9] Y. Tong, S. Bohm, M. Song, The capability of graphene on improving the electrical conductivity and anti-corrosion properties of polyurethane coatings, Appl. Surf. Sci. 424 (2017) 72-81.

[10] M. Mo, W. Zhao, Z. Chen, Q. Yu, Z. Zeng, X. Wu, Q. Xue, Excellent 
trobological and anti-corrosion performance of polyurethane composite coatings reinforced with functionalized graphene and graphene oxide nanosheets, RSC Adv. 5 (2015) 56486-56497.

[11] K. Cai, S. Zuo, S. Luo, C. Yao, W. Liu, J. Ma, H. Mao, Z. Li, Preparation of polyaniline/graphene composites with excellent anticorrosion properties and their application in waterborne polyurethane anticorrosive coatings, RSC Adv. 6 (2016) 95965-95972.

[12] W. Sun, L. Wang, T. Wu, Y. Pan, G. Liu, Synthesis of low-electrical-conductivity graphene/pernigraniline composites and their application in corrosion protection, Carbon. 79 (2014) 605-614.

[13] A. Krishnamurthy, V. Gadhamshetty, R. Mukherjee, Z. Chen, W. Ren, H. Cheng, N. Koratkar, Passivation of microbial corrosion using a graphene coating, Carbon. 56 (2013) 45-49.

[14] Y. Li, Z. Yang, H. Qiu, Y. Dai, Q. Zheng, J. Li, J. Yang, Self-aligned graphene as anticorrosive barrier in waterborne polyurethane composite coatings, J. Mater. Chem. A. 2 (2014) 14139-14145.

[15] S. Liu, L. Gu, H. Zhao, J. Chen, H. Yu, Corrosion resistance of graphene-reinforced waterborne epoxy coatings, J. Mater. Sci. Technol. 32 (2016) $425-431$

[16] T. Ramanathan, A. Abdala, S. Stankovich, D. Dikin, M. Herrera-Alonso, P. Piner, D. Adamson, D. Adamson, H. Schniepp, X. Chen, R. Ruoff, S. Nguyen, I. Aksay, P. Prud' homme, L. Brinson, Functionalized graphene sheets for polymer nanocomposites, Nat. Nanotech. 3 (2008) 327-331.

[17] J. Zhu, Graphene production: new solutions to a new problem, Nat. Nanotech. 3 (2008) 528-529. 
[18] M. Nonahal, M. R. Saeb, S. H. Jafari, H. Rastin, H. A. Khonakdar, F. Najafi, F. Simon, Design, preparation, and characterization of fast cure epoxy/amine-functionalized graphene oxide nanocomposites, Polym. Composite. ( 2017) doi: 10.1002/pc.24415.

[19] E. Yarahmadi, K. Didehban, M.G. Sari, M.R. Saeb, M. Shabanian, F. Aryanasab, P. Zarrintaj, S. M. R. Paran, M. Mozafari, M. Rallini, D. Puglia, Development and curing potential of epoxy/starch-functionalized graphene oxide nanocomposite coatings, Prog. Org. Coat. 119 (2018) 194-202.

[20] Y. Li, S. Chai, W. Yao, S. Deng, Q. Fu, F. Chen, Improved noncovalent functionalization and aqueous dispersibility of graphene via removing carbonaceous adsorbates, Mater. Lett. 100 (2013) 207-211.

[21] C. Liu, P. Du, H. Zhao, L. Wang, Synthesis of L-Histidine-attached graphene nanomaterials and their application for steel protection, ACS Appl. Nano Mater. $1(2018)$ 1385-1395.

[22] J. Ding, O. Rahman, W. Peng, H. Dou, H. Yu, A novel hydroxyl epoxy phosphate monomer enhancing the anticorrosive performance of waterborne graphene/epoxy coatings, Appl. Surf. Sci. 47 (2018) 981-991.

[23] X. Wang, W. Xing, L. Song, H. Y. Yang, Y. Hua, H. Y. Guan, Fabrication and characterization of graphene-reinforced waterborne polyurethane nanocomposite coatings by the sol-gel method, Surf. Coat. Technol. 206 (2012) 4778-4784.

[24] S. Qiu, G. Liu, W. Li, H. Zhao, L. Wang, Noncovalent exfoliation of graphene and its multifunctional composite coating with enhanced anticorrosion and tribological performance, J. Alloy. Compd. 747 (2018) 60-70.

[25] M. Mo, W. Zhao, Z. Chen, Q. Yu, Z. Zeng, X. Wu, Q. Xue, Excellent trobological and anti-corrosion performance of polyurethane composite coatings 
reinforced with functionalized graphene and graphene oxide nanosheets, RSC Adv. 5 (2015) 56486-56497.

[26] J. Li, J. Cui, J. Yang, Y. Li, H. Qiu, J. Yang, Reinforcement of graphene and its derivatives on the anticorrosive properties of waterborne polyurethane coatings, Compos. Sci. Technol. 129 (2016) 30-37.

[27] T. Ramanathan, A. A. Abdala, S. Stankovich, D.A. Dikin, M. Herrera-Alonso, R.D. Piner, D.H. Adamson, H.C. Schniepp, X. Chen, R. S. Ruoff, S. T. Nguyen, I. A. Aksay, R. K. Prud'homme, L.C. Brinson, Functionalized graphene sheets for polymer nanocomposites, Nature Nanotech. 3 (2018) 327-331.

[28] H. H. Wang, Y. K. Niu, G. Q. Fei, Y. D. Shen, J. Lan, In-situ polymerization, rheology, morphology and properties of stable alkoxysilane-functionalized poly (urethane-acrylate) microemulsion, Prog. Org. Coat. 99 (2016) 400-411.

[29] K. Zhu, X. R. Li, H. H. Wang, J. Y. Li, G. Q. Fei, Electrochemical and anti-corrosion behaviors of water dispersible graphene/acrylic modified alkyd resin latex composites coated carbon steel, J. Appl. Polym. Sci. 134 (2016) $44445-44456$.

[30] M. R. Sanchis, O. Calvo, O. Fenollar, D. Garcia, R. Balart, Characterization of the surface changes and the aging effects of low-pressure nitrogen plasma treatment in a polyurethane film, Polym. Test. 27 (2008) 75-83.

[31] S. Park, J. An, J. R. Potts, A. Velamakanni, S. Murali, R.S. Ruoff, Hydrazine-reduction of graphite- and graphene oxide, Carbon, 49 (2011) 3019-3023.

[32] X. Y. Zhang, H. P. Li , X. L. Cui , et al, Graphene/TiO 2 nanocomposites: synthesis, characterization and application in hydrogen evolution from water photocatalytic splitting, J. Mater. Chem. 20 ( 2010) 2801-2806. 
[33] G. Muruganandi, M. Saravanan, G. Vinitha, M. B. Jessie Raj, T. C. Sabari Girisum, Effect of reducing agents in tuning the third-order optical nonlinearity and optical limiting behavior of reduced graphene oxide, Chem. Phys. 488 (2017) $55-61$.

[34] D. Deng, X. Pan, H. Zhang, Q. Fu, D. Tan, X. Bao, Freestanding graphene by thermal splitting of silicon carbide granules, Adv. Mater. 22 (2010) 2168-2171.

[35] S. Stankovich, D. A. Dikin, R. D. Piner, K. A. Kohlhaas, A. Kleinhammes, Y. Jia, Y. Wu, S. T. Nguyen, R. S. Ruoff, Synthesis of graphene-based nanosheets via chemical reduction of exfoliated graphite oxide, Carbon. 45 (2007) 1558-1565.

[36] Y. Zhan, J. Zhang, X. Wan, Z. Long, S. He, Y. He, Epoxy composites coating with $\mathrm{Fe}_{3} \mathrm{O}_{4}$ decorated graphene oxide: Modified bio-inspired surface chemistry, synergistic effect and improved anti-corrosion performance, Appl. Surf. Sci. 436 (2018) 756-767.

[37] S. Pourhashem, M. R. Vaezi, A. Rashidi, M. R. Bagherzadeh, Exploring corrosion protection propertied of solvent based epoxy-graphene oxide nanocomposite coatings on mild steel, Corros. Sci. 115 (2017) 78-92.

[38] U. Stöberl, U. Wurstbauer, W. Wegscheider, D. Weiss, J. Ermos, Morphology and flexibility of graphene and few-layered graphene on various substrates. Appl. Phys, Lett. 939 (2008) 051906.

[39] D. Dutta, A. N. F. Ganda, J. Chih, C. Huang, C. Tseng, C. Su, Revisiting graphene-polymer nanocomposite for enhancing anticorrosion performance: a new insight into interface chemistry and diffusion model, Nanoscale. 10 (2018) $12612-12624$.

[40] B. Ramezanzaden, G. Bahlakeh, M. Ramezanzadeh, Polyaniline-cerium oxide (PAni- $\mathrm{CeO}_{2}$ ) coated graphene oxide for enhancement of epoxy coating corrosion 
protection performance on mild steel, Corros Sci. 137 (2018) 111-126. 